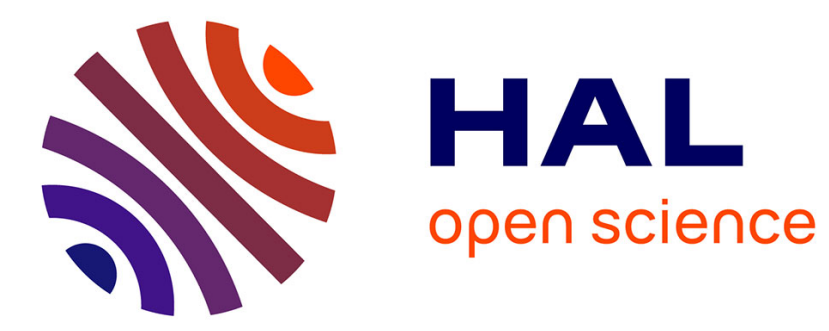

\title{
Water-waves as a spatial dynamical system
}

Frédéric Dias, Gérard Iooss

\section{To cite this version:}

Frédéric Dias, Gérard Iooss. Water-waves as a spatial dynamical system. S. Friedlander, D. Serre. Handbook of Mathematical Fluid Dynamics, Elsevier, pp.443-499, 2003. hal-00017484

\section{HAL Id: hal-00017484 https://hal.science/hal-00017484}

Submitted on 23 Jan 2006

HAL is a multi-disciplinary open access archive for the deposit and dissemination of scientific research documents, whether they are published or not. The documents may come from teaching and research institutions in France or abroad, or from public or private research centers.
L'archive ouverte pluridisciplinaire HAL, est destinée au dépôt et à la diffusion de documents scientifiques de niveau recherche, publiés ou non, émanant des établissements d'enseignement et de recherche français ou étrangers, des laboratoires publics ou privés. 


\title{
Water-waves as a spatial dynamical system
}

\author{
Frédéric Dias \& Gérard Iooss \\ F.D.: CMLA UMR CNRS 8536, ENS Cachan \\ 61 Ave du Président Wilson, F-94235 Cachan \\ e-mail:dias@cmla.ens-cachan.fr \\ G.I.: Institut Universitaire de France \\ INLN UMR CNRS-UNSA 6618 \\ 1361 route des Lucioles, F-06560 Valbonne
}

e-mail: iooss@inln.cnrs.fr

\begin{abstract}
The mathematical study of travelling waves, in the context of two dimensional potential flows in one or several layers of perfect fluid(s) and in the presence of free surface and interfaces, can be formulated as an ill-posed evolution problem, where the horizontal space variable plays the role of "time". In the finite depth case, the study of near equilibria waves reduces to a low dimensional reversible ordinary differential equation. In most cases, it appears that the problem is a perturbation of an integrable system, where all types of solutions are known. We describe the method of study and review typical results. In addition, we study the infinite depth limit, which is indeed a case of physical interest. In such a case, the above reduction technique fails because the linearized operator possesses an essential spectrum filling the whole real axis, and new adapted tools are necessary. We also discuss the latest results on the existence of travelling waves in stratified fluids and on three dimensional travelling waves, in the same spirit of reversible dynamical systems. Finally, we review the recent results on the classical two-dimensional standing wave problem.
\end{abstract}

\section{Contents}

2 Two-dimensional travelling water waves as a reversible dynamical system

2.1 Case of one layer with or without surface tension acting on the free surface $\ldots \ldots \ldots \ldots \ldots \ldots$

2.1.1 Case without surface tension $(b=0)$. . . . . . . . . 6

2.1 .2 Case with surface tension $(b \neq 0) \ldots \ldots$. . . . . . . 7

2.2 Case of a fluid layer below an elastic ice plate . . . . . . . . . . . 8 
2.3 Case of two layers without surface tension . . . . . . . . . . . . . 9

2.4 Case of one layer of infinite depth, with surface tension . . . . . . 11

2.5 Case of two layers, one being infinitely deep, without surface nor

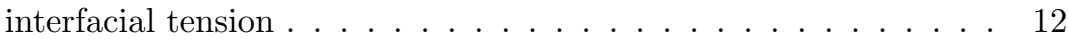

3 The linearized problem 13

3.1 Spectrum of the linearized operator . . . . . . . . . . . . . . . . 13

3.2 Physical situations $\ldots \ldots \ldots \ldots$. . . . . . . . . . . . . . . . . . . . . . . 17

3.3 Center manifold reduction (finite depth case) . . . . . . . . . . . 19

4 Finite depth case via reversible normal forms 20

4.1 Case of one layer without surface tension . . . . . . . . . . . . 20

4.2 Case of one layer with surface tension . . . . . . . . . . . . 24

4.2.1 Case (i): $0^{2+}$ resonance . . . . . . . . . . . . . . . . 24

4.2 .2 Case (ii): $0^{2+}(i q)$ resonance . . . . . . . . . . . . . . . . 25

$4.2 .3 \quad$ Case (iii): $(i q)^{2}$ resonance . . . . . . . . . . . . . . . . . . 29

4.2.4 Bifurcation of plethora of solitary waves . . . . . . . . . . 32

4.3 Case of two layers without surface tension . . . . . . . . . . . . 34

5 Case of infinite depth 35

5.1 Periodic waves . . . . . . . . . . . . . . . . . . . . . 36

5.2 Normal forms in infinite dimensions . . . . . . . . . . . . . . . . 38

5.3 Results for the $(i q)^{2}$ resonance with continuous spectrum . . . . 40

5.4 A new reversible bifurcation: pair of eigenvalues diving in the essential spectrum through the origin . . . . . . . . . . 44

$\begin{array}{lll}6 & \text { Stratified fluids } & 42\end{array}$

7 Three-dimensional travelling water waves 43

7.1 Formulation as a dynamical system . . . . . . . . . . . . . . . . 44

7.2 Spectrum of the linearized operator . . . . . . . . . . . . . . 47

7.3 Three-dimensional travelling waves periodic in the direction of

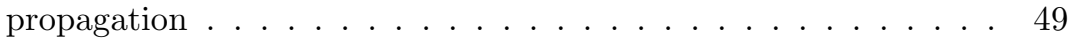

7.4 Dimension breaking bifurcation . . . . . . . . . . . . . . . . . 49

8 Two-dimensional standing wave problem 50 


\section{Introduction}

The present article focuses on classical problems in the theory of water waves. The topic of water waves is an old one and one can say that the theory of water waves was initiated by Stokes [108] in 1847. What do we mean by the classical problem of water waves? We mean the problem consisting in solving the incompressible Euler equations in a domain bounded above by a free surface (the interface between air and water) and below by a solid boundary (the bottom). The bottom can be at any depth (even infinite). The driving force is due to gravity. The effects of surface tension might be equally important and can be included in the analysis. There may be several superposed layers of immiscible fluids, with free interfaces between them, and with or without interfacial tension there. What makes the water-wave problem so difficult is not its governing equation which is linear (Laplace's equation), but its two nonlinear boundary conditions on each free surface and interface. For a lot of coastal engineering applications, solutions given by the linearized water-wave problem are accurate enough (see 105), but for a number of practical applications the fully nonlinear problem must be solved. Moreover, the water-wave problem has attracted mathematicians for almost a century because of its extremely rich structure.

It is important to emphasize here that the present review is restricted to a mathematical point of view. The most recent reviews on water waves, which are less mathematical, are those of Hammack \& Henderson [49] on resonant interactions among surface water waves, Banner \& Peregrine 12 on wave breaking in deep water, Dias \& Kharif [36] on the bifurcation, stability and evolution of water waves, Perlin \& Schultz [98] on capillary effects on surface waves, Duncan [39] on spilling breakers. Recent reviews on numerical aspects are those of Tsai \& Yue [117] and Scardovelli \& Zaleski [102] on the direct numerical simulation of free-surface and interfacial flows. For a complete bibliography on the numerical computation of three-dimensional water waves, one can refer to the paper [44.

Our review is more in the spirit of the section entitled "Existence theorems" in Wehausen \& Laitone's contribution to the Encyclopedia of Physics [120. Since the water-wave problem is a difficult nonlinear problem to solve, approximate theories have been developed. Most of these approximate theories are based on perturbation expansions and today perturbation expansions are still commonly used. When such approximate theories are used, it is tacitly assumed that there is an exact solution which is being approximated. Therefore existence and uniqueness proofs are an essential part of exact water-wave theory. But such proofs have generally been difficult to establish, and have usually been obtained for only rather restricted, although physically important, situations. No attempt will be made to give an exposition of all mathematical methods which have been used in establishing the various existing theorems.

Instead the purpose of the present review is to show how dynamical systems methods can be used to obtain results on the spatial behavior of travelling waves near the basic undisturbed free surface state. The water-wave problem can be viewed as a bifurcation problem which overlaps several important subjects: i) elliptic partial differential equations in unbounded domains like strips, ii) the 
theory of reversible systems in infinite dimensions, iii) the normal form technique and iv) the methods of analysis available for systems close to integrable ones. The idea to use "dynamical" arguments for solving nonlinear elliptic problems in a strip was developed in the 1980s, pioneered by K.Kirchgassner [73] (see [74] for a review on the water-wave problem).

We said above that our point of view is mathematical. But even within this point of view, our review is rather restricted. Indeed only dynamical systems aspects of the "small solutions" of the steady water-wave problem are considered, except for the section on standing waves, where the flow is unsteady. When dealing with progressive waves, we do not review the following aspects:

- Existence proofs based on methods of local analysis of the type of implicit functions theorem, including conformal mappings for reducing the problem to one of existence of a harmonic function satisfying nonlinear boundary conditions (see for example 81 and 94]), integral equation formulation (see for example [43]), Lyapunov-Schmidt method (see for example [29]);

- Existence proofs based on variational formulations (see for example [6], 48]);

- Mathematical results on approximate models or amplitude equations for water waves (see for example [18] for a review on model equations);

- Results on large-amplitude waves (see for example [7]), and results which rely on numerical arguments;

- Global results (see for example [77], [116], [5]);

- Stability results (see for example [88, 21]);

- Existence (or non-existence) of solutions to the Cauchy problem for water waves (see the work of $\mathrm{Wu}[122],[123]$ ). This is a still widely open problem. Existing results are valid on a finite (small) time interval and impose restrictions either on the size or on the smoothness of the initial data;

- Fully rigorous derivations of nonlinear amplitude equations (see for example 103 ).

Finally we deliberately present the problem in the framework of reversible vector fields, thus not taking advantage of the Hamiltonian structure of the spatial water-wave problem. This Hamiltonian structure has been fully justified and is more and more used in the mathematical theory of water waves (see for example [47, 48] and their bibliography). Our point of view allows a simpler presentation and the mathematical arguments we provide can be used in more general settings than just the Hamiltonian ones. 


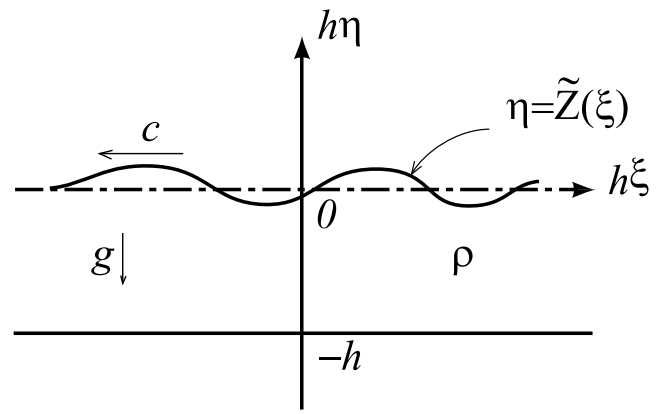

Figure 1: Sketch of a wave travelling along the free surface of a fluid layer of thickness $h$

\section{Two-dimensional travelling water waves as a reversible dynamical system}

\subsection{Case of one layer with or without surface tension act- ing on the free surface}

Consider first the case of one layer (thickness $h$ ) of an inviscid fluid of density $\rho$ under the influence of gravity $g$, with or without surface tension $T$ acting on the free surface (see figure 1). The flow is assumed to be potential. We are interested in steady waves of permanent form, i.e. travelling waves with constant velocity $c$. In a reference frame moving with the wave, these solutions are steady in time, and we intend to consider the unbounded horizontal coordinate $\xi$ as "time". Natural scales are $c$ for the velocity, $h$ for the length and $\rho c^{2}$ for the pressure. Once the equations are written in dimensionless form, two important parameters, one based on gravity and the other one on surface tension occur in the equations:

$$
\left.\left.\lambda=\frac{g h}{c^{2}} \text { (inverse of (Froude number }\right)^{2}\right) \text { and } \quad b=\frac{T}{\rho h c^{2}} \text { (Weber number). }
$$

The choice of scales is not unique. For example one could have chosen the capillary length $T /\left(\rho c^{2}\right)$ or the gravity length $c^{2} / g$ as length scale. What is important is that the equation depend only on two dimensionless parameters. In dimensionless form, the complex potential is denoted by $w(\xi+i \eta)$ and the complex velocity by $w^{\prime}(\xi+i \eta)=\underline{u}-i \underline{v}$. The free surface is denoted by $\eta=\widetilde{Z}(\xi)$. The Euler equations are expressed here by the fact that $w$ is analytic in $\zeta=\xi+i \eta$ and by the boundary condition

$$
\underline{v}=0 \text { at } \eta=-1 \text { (flat bottom), }
$$

the kinematic condition on the free surface

$$
\underline{u} \widetilde{Z}^{\prime}(\xi)-\underline{v}=0 \text { at } \eta=\widetilde{Z}(\xi) \text { (free surface), }
$$


and the Bernoulli first integral on the free surface, expressing the condition that the pressure jump is proportional to the curvature:

$$
\frac{1}{2}\left(\underline{u}^{2}+\underline{v}^{2}\right)+\lambda \widetilde{Z}-\frac{b \widetilde{Z}^{\prime \prime}}{\left(1+\widetilde{Z}^{\prime 2}\right)^{3 / 2}}=C \text { at } \eta=\widetilde{Z}(\xi) \text { (free surface) }
$$

where $C$ is a constant, and $b=0$ in the case of no surface tension. In order to formulate the problem as a dynamical system, we first transform the unknown domain into a strip. There are different ways for performing such a change of coordinates. We choose the one used by Levi-Civita 81.

Remark: We could have chosen another one, for instance the two-dimensional version of the change of coordinates used in section 7.1 for the three-dimensional case or the intermediate choice made in [74. The main advantage of the LeviCivita variables is that they lead to a weakly nonlinear problem, instead of a true quasi-linear partial differential equation (PDE) problem. Moreover a big part of the system stays linear, which is helpful.

The new unknown is $\alpha+i \beta$ as an analytic function of $w=x+i y$, where $x$ and $y$ are the potential and the stream function respectively, and

$$
w^{\prime}(\xi+i \eta)=e^{\beta-i \alpha} .
$$

The free surface is given by $y=0$ and the rigid bottom by $y=-1$. The function $\alpha$ represents the angle of the streamline with the horizontal, while $\beta$ represents the logarithm of the velocity modulus. Observe that fixing $y=0$ along the upper surface means that we impose the volume flux to be 1 in the moving frame, while we leave free the Bernoulli constant $C$.

Our formulation uses an unknown vector function denoted by $U$, which satisfies a differential equation of the form

$$
\frac{d U}{d x}=F(\mu, U)
$$

where $\mu$ represents the set of parameters.

\subsubsection{Case without surface tension $(b=0)$}

In such a case, $U$ in (11) is defined by

$$
[U(x)](y)=\left(\beta_{0}(x), \alpha(x, y), \beta(x, y)\right)^{t}
$$

where $\beta_{0}(x)=\beta(x, 0)$, while $\mu=\lambda$ and

$$
F(\lambda, U)=\left\{\begin{array}{c}
-\lambda e^{-3 \beta_{0}} \sin \alpha_{0} \\
\frac{\partial \beta}{\partial y} \\
-\frac{\partial \alpha}{\partial y}
\end{array}\right\}-1<y<0
$$

where $\alpha_{0}=\left.\alpha\right|_{y=0}$ and $\left.\alpha\right|_{y=-1}=0$. The first component of the system (11) is obtained after taking the derivative of the Bernoulli first integral with respect to 
$x$. It leads to an apparent loss of information, which in fact explains the arbitrary constant which appears in the analysis, corresponding to the Bernoulli constant. We observe that the free surface may be deduced from $U$ by the formula (which defines $Z(x))$

$$
\eta=\widetilde{Z}(\xi)=Z(x)=\int_{-1}^{0}\left(e^{-\beta} \cos \alpha-1\right) d y
$$

and

$$
\frac{d \xi}{d x}=e^{-\beta_{0}} \cos \alpha_{0}
$$

Equation (11) has to be understood in the Hilbert space $\mathbb{H}=\mathbb{R} \times\left\{L^{2}(-1,0)\right\}^{2}$, and $U(x)$ lies in $\mathbb{D}=\mathbb{R} \times\left\{H^{1}(-1,0)\right\}^{2} \cap\left\{\beta_{0}=\left.\beta\right|_{y=0},\left.\alpha\right|_{y=-1}=0\right\}$, where $H^{1}(-1,0)$ denotes the Sobolev space of square integrable functions with a square integrable first derivative on the interval $(-1,0)$. So, for a fixed $x$ the right hand side of (2) is a function of $y$ and $U(x)$ is required to satisfy the boundary conditions indicated in $\mathbb{D}$. A solution of the water-wave problem is any $U \in$ $\mathcal{C}^{0}(\mathbb{D}) \cap \mathcal{C}^{1}(\mathbb{H})$ which is solution of $(1)$, where (e.g.) $\mathcal{C}^{0}$ means continuous and bounded for $x \in \mathbb{R}$.

It is clear that $U=0$ is a particular solution of (11), which corresponds to the flat free surface state. Notice that we have a one parameter family of constant solutions $U=\left(\beta_{0}, 0, \beta_{0}\right)^{t}$ with arbitrary $\beta_{0}$. They give a flat free boundary $Z=e^{-\beta_{0}}-1(\neq 0$ in general $)$, and correspond to varying the Bernoulli constant C.

An important property of (11) is its reversibility. Indeed let us define the symmetry:

$$
S U=\left(\beta_{0},-\alpha, \beta\right)^{t} .
$$

It is easy to see that the linear operator $S$ anticommutes with $F(\lambda, \cdot)$. This reflects the invariance under reflexion symmetry $x \rightarrow-x$ of the problem.

\subsubsection{Case with surface tension $(b \neq 0)$}

In such a case, the variable $U$ in (11) is defined by

$$
[U(x)](y)=\left(\alpha_{0}(x), \alpha(x, y), \beta(x, y)\right)^{t}
$$

and

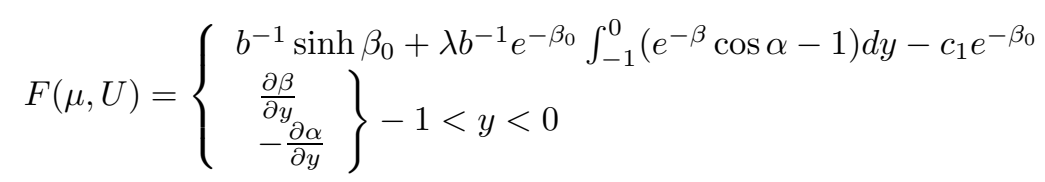

where $\mu=\left(\lambda, b, c_{1}\right), b c_{1}=C-1 / 2$ and $\beta_{0}=\beta(x, 0)$. Equation (11) has to be understood in the Hilbert space $\mathbb{H}=\mathbb{R} \times\left\{L^{2}(-1,0)\right\}^{2}$, and $U(x)$ lies in $\mathbb{D}=\mathbb{R} \times\left\{H^{1}(-1,0)\right\}^{2} \cap\left\{\alpha_{0}=\left.\alpha\right|_{y=0},\left.\alpha\right|_{y=-1}=0\right\}$. The free surface is given by formula (3). Here again $U=0$ is a particular solution of (11) for $c_{1}=0$, 
which corresponds to the flat free surface state and (1) is reversible under the symmetry defined by:

$$
S U=\left(-\alpha_{0},-\alpha, \beta\right)^{t} .
$$

Notice that for $c_{1}>-\frac{1}{2 b}\left(1+2 \lambda-3 \lambda^{2 / 3}\right)$, there are two families of "conjugate" constant solutions $U_{c_{1}}=\left(0,0, \beta_{0}\right)^{t}$, where

$$
\left(1-e^{\beta_{0}}\right)\left[\lambda e^{-\beta_{0}}-1 / 2\left(1+e^{\beta_{0}}\right)\right]=b c_{1} .
$$

These constant solutions are such that the free surface is flat and $Z=e^{-\beta_{0}}-1$ ( $\neq 0$ in general). Fixing $c_{1}=0$ would mean that the velocity scale $c$ we chose a priori corresponds to $\beta_{0}=0$, i.e. $U=0$, while the conjugate flow has a different velocity given by $e^{\beta_{0}}=2^{-1}\left[(1+8 \lambda)^{1 / 2}-1\right]$. Since we only study solutions of equation (1) near $U=0$, the occurence of this conjugate flow would then only matter when $\lambda$ is close to 1 . This means that for $\lambda$ close to $1, \lambda$ is not well defined, since we arbitrarily choose one of the two conjugate flows for the choice of the velocity scale. This is why we prefer to keep $c_{1}$ as an additional parameter.

\subsection{Case of a fluid layer below an elastic ice plate}

This case takes place in particular when a relatively thin layer of ice covers a large lake. The equations are the same as in the case with surface tension, except for the dynamic condition on the free surface. Assuming that the terms involving the ice thickness can be neglected, using Bernoulli's equation and following the approach of Forbes [42] and Il'ichev \& Kirchgässner [55], who model the ice sheet as a Kirchhoff-Love plate, one finds the following dynamic condition

$$
\frac{1}{2}\left(\underline{u}^{2}+\underline{v}^{2}\right)+\lambda \tilde{Z}+D\left(\frac{\widetilde{Z}^{\prime \prime}}{\left(1+\tilde{Z}^{\prime 2}\right)^{3 / 2}}\right)^{\prime \prime}=C \quad \text { at } \quad \eta=\tilde{Z}(\xi) \quad \text { (interface) }
$$

where $C$ is a constant and $D$ is a dimensionless parameter. It is given by

$$
D=\left(\rho c^{2} h^{3}\right)^{-1} \frac{E h_{i}^{3}}{12\left(1-\nu^{2}\right)}
$$

where $E$ is the Young's modulus of the ice, $h_{i}$ the ice thickness and $\nu$ the Poisson's ratio for ice. The scales for length, pressure and velocity are the same as before.

Again one can introduce the change of coordinates used by Levi-Civita

$$
w^{\prime}(\xi+i \eta)=e^{\beta-i \alpha} .
$$

The interface is given by $y=0$ and the rigid bottom by $y=-1$. The variable $U$ in (1) is defined by

$$
[U(x)](y)=\left(\alpha_{0}(x), V(x), W(x), \alpha(x, y), \beta(x, y)\right)^{t}
$$




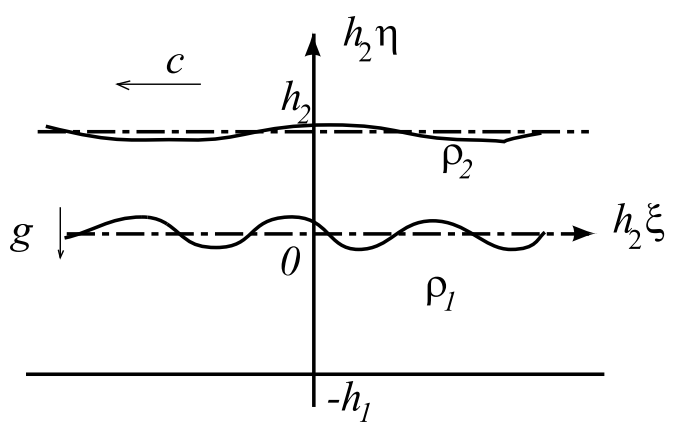

Figure 2: Two superposed fluid layers of finite depth

and

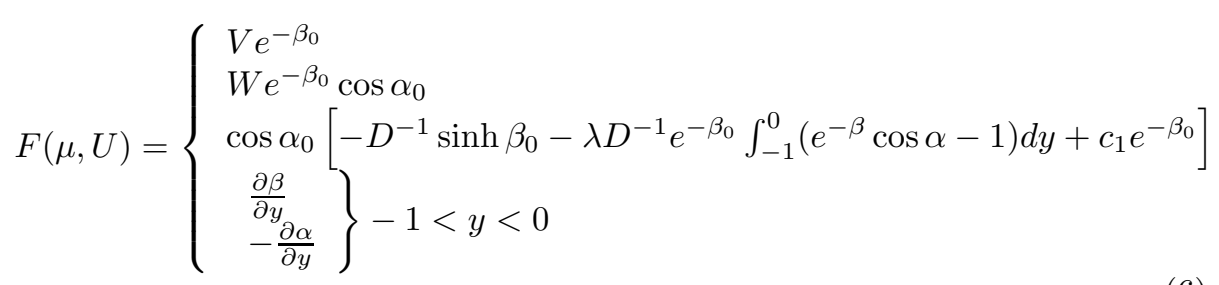

where $\mu=\left(\lambda, D, c_{1}\right), D c_{1}=C-1 / 2$ and $\beta_{0}=\beta(x, 0)$. The boundary conditions for $U$ are $\alpha(x,-1)=0$ and $\alpha(x, 0)=\alpha_{0}(x)$. The interface is given by formula (3). Here again $U=0$ is a particular solution of (1) for $c_{1}=0$, which corresponds to the flat ice sheet and (1) is reversible under the symmetry defined by:

$$
S U=\left(-\alpha_{0}, V,-W,-\alpha, \beta\right)^{t} .
$$

The system (1, 6) has to be understood in $\mathbb{H}=\mathbb{R}^{3} \times\left[L^{2}(-1,0)\right]^{2}$, and $U(x)$ lies in

$$
\mathbb{D}=\mathbb{R}^{3} \times\left[H^{1}(-1,0)\right]^{2} \cap\left\{\alpha_{0}=\left.\alpha\right|_{y=0},\left.\alpha\right|_{y=-1}=0\right\} .
$$

\subsection{Case of two layers without surface tension}

Let us now consider the two-layer case (densities $\rho_{1}, \rho_{2}$ ), assuming that there is no surface tension, neither at the free surface nor at the interface. At rest, the thickness of the upper layer is $h_{2}$ while it is $h_{1}$ for the bottom one (see figure 2). The dimensionless parameters we find convenient are $\rho=\rho_{2} / \rho_{1}<1, e=h_{1} / h_{2}$, and $\lambda=\frac{g h_{2}}{c^{2}}$. The domain can be transformed into two superposed horizontal strips and we may use the same type of variables as above, except that now the length scale is $h_{2}$. We use $h_{2}$ as length scale in order to be able to take the limit $h_{1} \rightarrow \infty$ below. One difficulty is that the $x$ coordinate is not the same in each strip! We choose as the basic $x$ coordinate the one given by the bottom layer. This choice introduces a factor in the Cauchy-Riemann equations of the 
upper layer since $\frac{d x_{2}}{d x_{1}}=e^{\beta_{20}-\beta_{10}}$, where $\beta_{j 0}$ is the trace of $\beta_{j}$ at $y=0$. In such a formulation, the unknown is defined by

$$
[U(x)](y)=\left(\beta_{10}(x), \beta_{21}(x), \alpha_{1}(x, y), \beta_{1}(x, y), \alpha_{2}(x, y), \beta_{2}(x, y)\right)^{t}
$$

and the system has the form (11) with $\mu=(\rho, e, \lambda)$ and

$$
F(\mu, U)=\left\{\begin{array}{cc}
-\lambda(1-\rho) \sin \alpha_{10} e^{-3 \beta_{10}}-\left.\rho e^{3\left(\beta_{20}-\beta_{10}\right)} \frac{\partial \alpha_{2}}{\partial y}\right|_{y=0} \\
-\lambda \sin \alpha_{21} e^{-3 \beta_{21}+\beta_{20}-\beta_{10}} \\
\left.\begin{array}{cc}
\frac{\partial \beta_{1}}{\partial y} \\
-\frac{\partial \alpha_{1}}{\partial y}
\end{array}\right\} & -e<y<0 \\
\frac{\partial \beta_{2}}{\partial y} e^{\beta_{20}-\beta_{10}} \\
-\frac{\partial \alpha_{2}}{\partial y} e^{\beta_{20}-\beta_{10}}
\end{array}\right\} \quad \begin{gathered}
0<y<1
\end{gathered}
$$

where we denote by $\alpha_{10}$ the trace of $\alpha_{1}$ at $y=0$, and by $\alpha_{21}, \beta_{21}$ the traces of $\alpha_{2}$ and $\beta_{2}$ at $y=1$. Let $W^{p, 1}(I)$ be the Sobolev space of integrable functions on the interval $I$, having integrable derivatives up to $p t h$ order on this interval. The basic Banach space $\mathbb{H}=\mathbb{R}^{2} \times\left\{W^{1,1}(-e, 0)\right\}^{2} \times\left\{W^{1,1}(0,1)\right\}^{2}$ requires more regularity than in the previous case, in order to be able to define the term $\left.\frac{\partial \alpha_{2}}{\partial y}\right|_{y=0}$ in the first component and the domain of the vector field $F$ is now:

$$
\begin{aligned}
\mathbb{D} & =\mathbb{R}^{2} \times\left\{W^{2,1}(-e, 0)\right\}^{2} \times\left\{W^{2,1}(0,1)\right\}^{2} \cap \\
\cap\left\{\alpha_{10}\right. & \left.=\alpha_{20}, \beta_{10}=\left.\beta_{1}\right|_{y=0}, \beta_{21}=\left.\beta_{2}\right|_{y=1},\left.\alpha_{1}\right|_{y=-e}=0\right\},
\end{aligned}
$$

while the reversibility symmetry reads:

$$
S U=\left(\beta_{10}, \beta_{21},-\alpha_{1}, \beta_{1},-\alpha_{2}, \beta_{2}\right)^{t} .
$$

Notice that we do not use here Hilbert spaces: this is precisely due to the "bad" trace term $\left.\frac{\partial \alpha_{2}}{\partial y}\right|_{y=0}$ in the first component of the vector field (7). Another choice of space, such as $H^{1}$ instead of $W^{1,1}$, would not lead to a good estimate of the resolvent operator (see below). The new system (1, 7) must be completed by the following two Bernoulli first integrals (interface and free surface):

$$
\begin{aligned}
& \lambda(1-\rho) \int_{-e}^{0}\left(e^{-\beta_{1}} \cos \alpha_{1}-1\right) d y+1 / 2\left(e^{2 \beta_{10}}-1\right)-\rho / 2\left(e^{2 \beta_{20}}-1\right)=C_{1} \\
& \int_{-e}^{0}\left(e^{-\beta_{1}} \cos \alpha_{1}-1\right) d y+\int_{0}^{1}\left(e^{-\beta_{2}} \cos \alpha_{2}-1\right) d y+1 / 2 \lambda\left(e^{2 \beta_{21}}-1\right)=C_{2}
\end{aligned}
$$

which give the first two components of (11, 17) after differentiation. In principle we might choose to treat this problem on a codimension- 2 manifold, instead of expressing these first two components. It appears that it is easier to work as we do if we keep in mind that there are two known first integrals. This freedom on the choice of the two Bernoulli constants is due to the fact that the system (1, ,7) has a two-parameter family of constant solutions $U=\left(\beta_{10}, \beta_{21}, 0, \beta_{10}, 0, \beta_{21}\right)^{t}$ corresponding to uniform flows in each layer, with different velocities. 


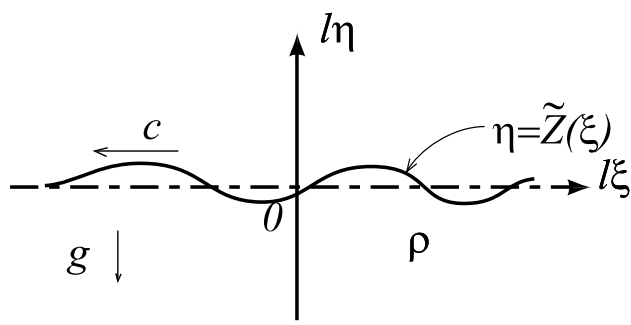

Figure 3: One layer of infinite depth

\subsection{Case of one layer of infinite depth, with surface ten- sion}

Let us now consider the case of one infinitely thick layer of an inviscid fluid. The flow is still assumed to be potential (see figure 3). The notation is the same as for the finite depth case, except that we choose a new length scale $l=T / \rho c^{2}$. The dimensionless parameter occuring in the equations now is

$$
\mu_{1}=\frac{g T}{\rho c^{4}} .
$$

The free surface is denoted by $\eta=\widetilde{Z}(\xi)$, and we have the boundary conditions

$$
(\underline{u}, \underline{v}) \rightarrow(1,0) \text { as } \eta \rightarrow-\infty,
$$

and the same conditions (kinematic and dynamic) on the free surface as for the one-layer case treated above.

Using again the coordinates of Levi-Civita 81, the free surface is given by $y=0$, and the flow region by $y<0$. In our formulation, the variable $U$ is defined by

$$
[U(x)](y)=\left(Z(x), \alpha_{0}(x), \alpha(x, y), \beta(x, y)\right)^{t}
$$

and it is straightforward to obtain the system under the form (1), with

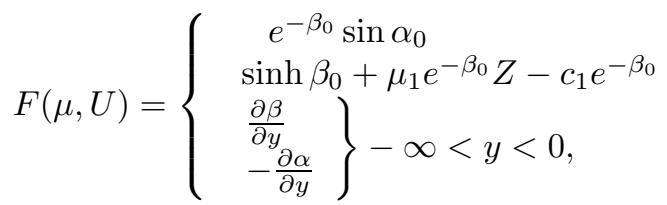

where $\mu=\left(\mu_{1}, c_{1}\right)$ and $c_{1}$ is the Bernoulli constant. The system (11.9) has to be understood in the space $\mathbb{H}=\mathbb{R}^{2} \times\left\{L^{1}\left(\mathbb{R}^{-}\right)\right\}^{2}$, and $U(x)$ lies in $\mathbb{D}=$ $\mathbb{R}^{2} \times\left\{W^{1,1}\left(\mathbb{R}^{-}\right)\right\}^{2} \cap\left\{\alpha_{0}=\left.\alpha\right|_{y=0}\right\}$. As above, a solution of the water-wave problem is any $U \in \mathcal{C}^{0}(\mathbb{D}) \cap \mathcal{C}^{1}(\mathbb{H})$ which is solution of (1, 99).

It is clear that $U=0$ is a particular solution of (1.9) for $c_{1}=0$, which corresponds to the flat free surface state, and the system (1.1.9) is again reversible, under the symmetry

$$
S U=\left(Z,-\alpha_{0},-\alpha, \beta\right)^{t} .
$$


Note that in the unbounded strip $y<0$ the system (1, 19 is in linear: This is very helpful for the study of solitary waves (see below and [65]) because it makes the choice of function space easier when the behavior at infinity in the $x$-coordinate has to be mixed with the behavior at infinity in the $y$-coordinate. Another remark for the one-layer case is that the problem can be reformulated into an integro-differential equation (after solving the linear problem in the region $y<0)$. However, one needs to specify from the beginning what type of solution is studied, and the choice of solutions is then restricted a priori. For instance, for periodic solutions one might use Fourier series spaces (see for instance [71]), and for solitary waves one might use a Hilbert transform in the half space (see a remark in 650). This possible reduction is not used here, since we want to apply our method to more general problems and solutions, especially to problems with several layers.

Remark: We can consider the above case as the limit of the case of one layer as the depth tends to infinity. For that it is necessary to write the system (1, 4) with the length scale $l$ (instead of $h$ ). The parameters now are $\mu_{1}$ and $b$. This modifies the vector field (4); in particular the strip $(-1,0)$ is replaced by $(-1 / b, 0)$, and $b \rightarrow 0$ as $h \rightarrow \infty$.

\subsection{Case of two layers, one being infinitely deep, without surface nor interfacial tension}

We go back to the case with two superposed immiscible fluid layers, without surface nor interfacial tension, but we assume now that the bottom layer is infinitely deep, i.e. $e=\infty$ (see figure 4 ). The formulation is then identical to the one above with $\mu=(\rho, \lambda)$ and the interval $(-e, 0)$ replaced by $\mathbb{R}^{-}$. The domain of the vector field is now defined by

$$
\begin{aligned}
\mathbb{D} & =\mathbb{R}^{2} \times\left\{W^{2,1}\left(\mathbb{R}^{-}\right)\right\}^{2} \times\left\{W^{2,1}(0,1)\right\}^{2} \cap \\
\cap\left\{\alpha_{10}\right. & \left.=\alpha_{20}, \beta_{10}=\left.\beta\right|_{y=0}, \beta_{21}=\left.\beta\right|_{y=1}\right\},
\end{aligned}
$$

and the new vector field is denoted by $\left(\mathbb{7}_{\infty}\right)$. However, even though there are two arbitrary Bernoulli constants, there is only a one-parameter family $U=\left(0, \beta_{21}, 0,0,0, \beta_{21}\right)^{t}$ of constant solutions. This is due to the fact that we implicitly impose the boundary conditions $\alpha_{1}$ and $\beta_{1} \rightarrow 0$ as $y \rightarrow-\infty$.

Remark: The case of more than two superposed fluid layers, with or without surface and interfacial tensions, may be easily formulated in the same way (see for instance $[58$ for two layers and various surface and interfacial conditions).

Finally, it has to be understood that problem (11) is not a usual evolution problem: the initial value problem is ill-posed! This is in fact an elliptic problem in the strip $\mathbb{R} \times(-1,0)$ for problems (1, 2), (1), 1 , , (1), 6), $\mathbb{R} \times(-e, 1)$ for problem (11.7), $\mathbb{R} \times \mathbb{R}^{-}$for problem (1, 9), $\mathbb{R} \times(-\infty, 1)$ for problem 1,1,$\left.]_{\infty}\right)$. However we treat this problem by local techniques of dynamical systems theory, following the idea introduced by Kirchgässner in [73]. In the finite depth case, the key feature is the possibility to apply a center manifold reduction into a reversible ordinary differential equation for the study of solutions staying close to 0 . In the infinite 


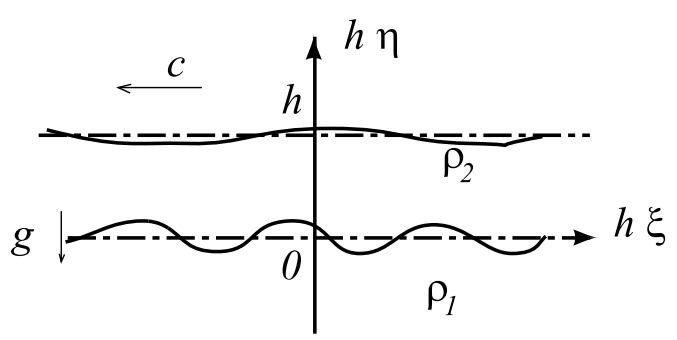

Figure 4: Two layers, one being infinitely deep

depth case, we are still able to isolate a finite dimensional-like family of solutions, whose behavior as $|x| \rightarrow \infty$ is different from the corresponding behavior in the finite depth case. Moreover, this introduces new types of bifurcations, not governed by a reduced finite dimensional reversible ordinary differential equation (ODE).

The aim of the next section is to show the properties of the linearized operator near 0 , which clarify the study of local solutions in all cases and, in particular, allow the use of such a center manifold reduction in the finite depth case.

\section{The linearized problem}

\subsection{Spectrum of the linearized operator}

Since we are interested in solutions near 0 , it is natural to study the linearized problem near 0 . This linearized system reads

$$
\frac{d U}{d x}=L_{\mu} U
$$

in $\mathbb{H}$. In all problems with finite-depth layers, it can be shown that the spectrum of the unbounded (closed) linear operator $L_{\mu}$ which is symmetric with respect to both axes of the complex plane because of reversibility, is only composed of isolated eigenvalues of finite multiplicities, only accumulating at infinity. More precisely, denoting by $i k$ these eigenvalues (not necessarily purely imaginary), then one has the classical "dispersion relation" satisfied by the eigenvalues, under the form of a complex equation $\Delta(\mu, k)=0$, analytic in $(\mu, k)$. The linearization of the vector fields (2, 4, 6, ,7) leads to the following dispersion relations, respectively:

$$
k-\lambda \tanh k=0,
$$

$$
\begin{aligned}
& k-\left(\lambda+b k^{2}\right) \tanh k=0, \text { for } k \neq 0, \\
& k-\left(\lambda+D k^{4}\right) \tanh k=0, \text { for } k \neq 0,
\end{aligned}
$$




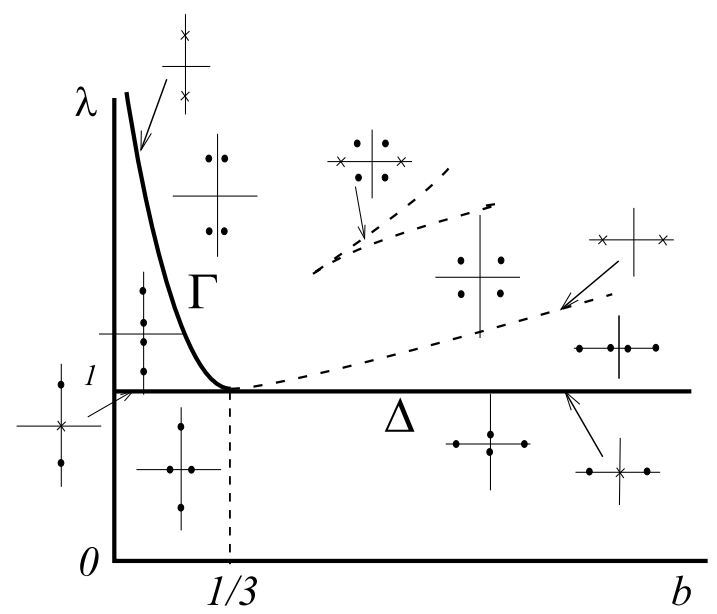

Figure 5: Position of the four "critical" eigenvalues $i k$, close to the imaginary axis, for the vector field linearized from (4), depending on the parameter values $(b, \lambda)$. Dashed curves correspond to the existence of double nonzero real eigenvalues.

$$
\rho\left(\lambda^{2}-k^{2}\right) \tanh (k e) \tanh k-(k-\lambda \tanh k)[k-\lambda \tanh (k e)]=0 .
$$

Note that we take $c_{1}=0$ for linearizing (4) and (6) since $c_{1}$ plays the role of an additional parameter to be considered later. Any real solution $k$ of $\Delta(\mu, k)=0$ gives a pure imaginary eigenvalue $i k$ of the linear operator $L_{\mu}$.

For a reason that we explain later, we are especially interested in eigenvalues which lie near or on the imaginary axis. Indeed, there is only a small number of eigenvalues on (or close to) the imaginary axis, the rest of them being located in a sector $\left(i k \in \mathbb{C} ;\left|k_{r}\right|<p\left|k_{i}\right|+r\right)$ of the complex plane.

For the system (11:2) $\Delta(\mu, k)=0$ is given by (11) and we have the following situation: for $\lambda<1,0$ is the only eigenvalue (simple) on the imaginary axis, for $\lambda>1$, we have 0 and a pair of simple eigenvalues lying on the imaginary axis, and for $\lambda=1,0$ is a triple eigenvalue. All other eigenvalues are not close to the imaginary axis, except for $\lambda$ near $1^{-}$where two symmetric real eigenvalues tend towards 0 as $\lambda \rightarrow 1$.

For the system (1, A $) \Delta(\mu, k)=0$ is given by $(12)$ and the eigenvalues close to the imaginary axis are described in figure 5 (see [74). The left side of the curve $\Gamma$ and the line $\Delta(\lambda=1)$ in the parameter plane $(b, \lambda)$ correspond to the occurence of double eigenvalues on the imaginary axis or at the origin, while the point $(b, \lambda)=(1 / 3,1)$ corresponds to the occurence of a quadruple eigenvalue 0 . The dashed curves correspond to the occurence of pairs of double real eigenvalues, the most important being the curve the closest to $\Delta$ (see section 4.2.4). 
For the system (1, 6) $\Delta(\mu, k)=0$ is given by (13) and the positions of the "critical" eigenvalues are described in figure 6. The curve $\Gamma$ in the parameter plane $(D, \lambda)(D$ is defined in section 2.2) corresponds to a pair of double eigenvalues on the imaginary axis (this pair tends towards 0 , when $D \rightarrow \infty$ ), while the line $\Delta(\lambda=1)$ corresponds to a double eigenvalue at the origin, in addition to a pair of simple eigenvalues on the imaginary axis, tending towards 0 as $D \rightarrow \infty$.

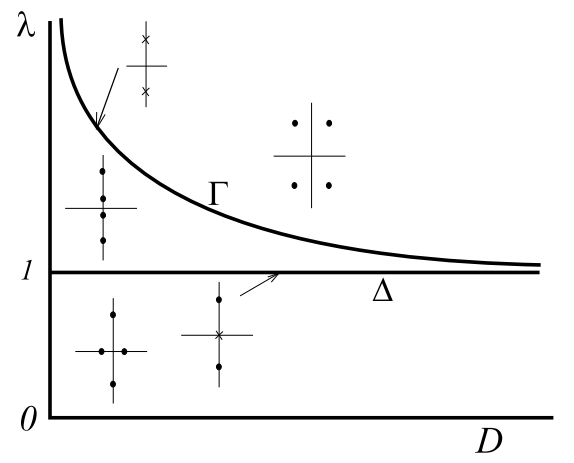

Figure 6: Position of the four critical eigenvalues $i k$, close to the imaginary axis, for the system linearized from (6), depending on the parameter values $(D, \lambda)$.

For the system (朋) $\Delta(\mu, k)=0$ is given by (14) and 0 is always a double eigenvalue, except on the set given by

$$
\lambda=\lambda_{0}^{ \pm}(e, \rho) \stackrel{\text { def }}{=}[2 e(1-\rho)]^{-1}\left[1+e \pm \sqrt{(e-1)^{2}+4 e \rho}\right],
$$

where 0 is a quadruple eigenvalue. The positions of the critical eigenvalues are shown in figure 7 .

We observe that 0 is always an eigenvalue for the linearized vector fields (2) and (7). This is due to the freedom in the choice of the Bernoulli constant, while for the fields (4) and (6) this happens only if $\lambda=1$ (we fixed the constant $\left.c_{1}=0\right)$.

The roots $k$ of the dispersion equations $\Delta(\mu, k)=0$ give the poles $\sigma=i k$ of the resolvent operator $\left(\sigma \mathbb{I}-L_{\mu}\right)^{-1}$.

In the case of an infinitely deep layer, for vector fields $L_{\mu} U$ linearized from (9) and (79 $)$ where $c_{1}=0$ in (9), the spectrum of $L_{\mu}$ is as follows (see the proofs in [65] and [58]):

i) there is a discrete set of isolated eigenvalues $i k$ of finite multiplicities, which are given by the roots of the dispersion relation $\Delta(\mu, \operatorname{sgn}(\operatorname{Re} k) k)=0$;

ii) the entire real axis constitutes the "essential spectrum". Moreover, 0 is an eigenvalue embedded in this continuous part of the spectrum for $\left(7_{\infty}\right)$. 


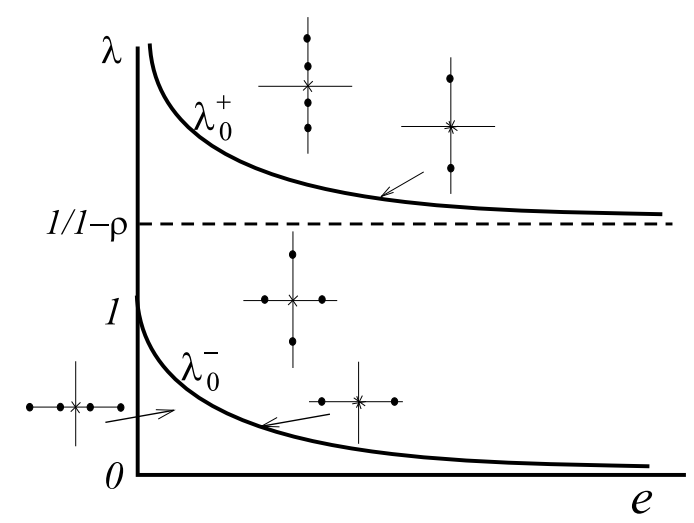

Figure 7: Position of the critical eigenvalues for the system linearized from (7), depending on the parameters $(e, \lambda)$ for a fixed value of $\rho$. The eigenvalue 0 is at least double, it is quadruple on the curves $\lambda_{0}^{ \pm}$.

More precisely, for (9) and $\left(77_{\infty}\right)$, respectively, we have for $\operatorname{Re} k>0$

$$
\begin{aligned}
& \Delta(\mu, k)=k^{2}-k+\mu_{1}, \\
& \Delta(\mu, k)=(\lambda-k)\{[\rho(\lambda+k)-\lambda] \tanh k+k\},
\end{aligned}
$$

which should be completed for $\operatorname{Re} k<0$ by $\Delta(\mu,-k)$ in order to obtain the symmetric spectrum.

The roots $k$ of the dispersion equations $\Delta(\mu, \operatorname{sgn}(\operatorname{Re} k) k)=0$ which are not purely imaginary or 0 give the poles $\sigma=i k$ of the resolvent operator $\left(\sigma \mathbb{I}-L_{\mu}\right)^{-1}$. The nature of the point 0 of the spectrum is more delicate. When 0 is not an eigenvalue, the structure of the resolvent operator near 0 is the same as near the rest of the real line; in particular, the linear operator $\left(\sigma \mathbb{I}-L_{\mu}\right)$ for $\sigma$ real has a non closed range, and the closure of the range has a codimension one for (9). When 0 is a simple eigenvalue, as for $\left(77_{\infty}\right)$, we are able to build a projection operator (on the one dimensional kernel), commuting with $L_{\mu}$ and such that 0 is no longer an eigenvalue of $L_{\mu}$ in the complementary invariant subspace. We are thus coming back to the previous case (9) (see details in [58]).

Some natural questions arise: what really happens in the spectrum for the vector fields (बi and (7), when the bottom layer thickness grows towards infinity? What are the physically realistic cases?

It is not difficult to see that as the depth is growing, there are more and more real eigenvalues accumulating regularly on the whole real axis, the eigenvectors being bounded, but not tending towards 0 . In the limit, as we choose a basic space such that $(\alpha, \beta) \rightarrow 0$ as $y \rightarrow-\infty$, all real eigenvalues disappear [except 0 for $\left(\nabla_{\infty}\right)$ ] but the spectrum stays as an essential spectrum. Concerning the physical relevance of studying the infinite depth case, we need to consider what are the characteristic scales of the problems. 


\subsection{Physical situations}

In this section we consider typical physical situations, in connection with the theoretical results we mention in this paper. All waves are travelling in the $\xi$ physical direction with a constant velocity $c$. We mention periodic waves, which are indeed periodic in $\xi$, solitary waves which are waves localized in space, i.e. tending to a flat pattern at infinity, and generalized solitary waves which are a sort of superposition of a periodic wave at infinity, and of a localized wave at finite distance.

In the following examples we take a typical value of surface tension for an air-water interface: $T / \rho=74 \mathrm{~cm}^{3} / \mathrm{s}^{2}$.

Cases in the frame of system (1, 2).

i) Tsunami: $h=4000 \mathrm{~m}$ (depth), $L=100 \mathrm{~km}$ (wave length). Hence $k=0.25$, and the corresponding value of $\lambda$ given by linear theory (11) is $\lambda=k / \tanh k \simeq 1$. Note that (11) corresponds to $b=0$, which is a good approximation since here $b=O\left(10^{-13}\right)$. Solving for $c$ gives $c=195 \mathrm{~m} / \mathrm{s}$ and we are close to a solitary wave generation.

ii) Solitary waves in a wave tank: in this case, $h=10 \mathrm{~cm}, L=250 \mathrm{~cm}$, hence $k=0.25, \lambda \simeq 1$ (as above), and this corresponds to $c=98 \mathrm{~cm} / \mathrm{s}$, while $b=O\left(10^{-4}\right)$.

iii) Wind waves, generated by a storm in the ocean: $h=1000 \mathrm{~m}, L=150 \mathrm{~m}$, hence $k=42$, and the dispersion relation gives $\lambda \sim k$, corresponding to $c=$ $15.2 \mathrm{~m} / \mathrm{s}$, while $b=O\left(10^{-10}\right)$. We are then far from the solitary wave generation.

Cases in the frame of systems (1, 4, or 1,9).

Here we note that fixing $h$ fixes the ratio $b / \lambda$ since $b / \lambda=T / \rho g h^{2}$. Then, once $k$ is known, the dispersion equation (12) gives $\lambda$ and $b$, and the corresponding velocity $c$ of the waves.

The dispersion relation (12) can be rewritten in physical variables as

$$
c^{2}=\left(\frac{g L}{2 \pi}\right) \tanh \left(\frac{2 \pi h}{L}\right)\left[1+\frac{b}{\lambda}\left(\frac{2 \pi h}{L}\right)^{2}\right] .
$$

For realistic water depths, the plot $c(L)$ exibits a minimum, say at $L=L^{*}$. Waves with $L<L^{*}$ are usually referred to as capillary waves, while waves with $L>L^{*}$ are referred to as gravity waves. For example, for $h=3 \mathrm{~cm}$ (and consequently $b / \lambda=0.0084$ ), one finds that $L^{*} \approx 1.7 \mathrm{~cm}$. Consequently, using the superscript $*$ to denote values at $L=L^{*}$, we have $k^{*}=11$, and $\lambda^{*} \sim 5.5$, $b^{*} \sim 0.046, c^{*}=23.2 \mathrm{~cm} / \mathrm{s}$. This point represents in fact the occurence of double imaginary eigenvalues and belongs to the curve $\Gamma$ in figure 5 .

On the capillary side, a typical value is $L=1 \mathrm{~cm}$. Hence $k=19$ and $\lambda \sim 4.7$, $b \sim 0.04, c=25 \mathrm{~cm} / \mathrm{s}$. The point in the parameter plane $(b, \lambda)$ is below the curve $\Gamma$.

On the gravity side, a typical value is $L=5 \mathrm{~cm}$. Hence $k=3.8$ and $\lambda \sim 3.4$, $b \sim 0.03, c=29.5 \mathrm{~cm} / \mathrm{s}$. The point in the parameter plane lies again below the curve $\Gamma$.

Note that although a depth of $3 \mathrm{~cm}$ may appear relatively shallow, it is in fact close to the deep water case introduced in Section 2.4 when one is interested in 
the $(i q)^{2}$ resonance (occurence of double imaginary eigenvalues)! In deep water, the $(i q)^{2}$ resonance occurs for a speed $c=23.2 \mathrm{~cm} / \mathrm{s}$ and a wavelength $L=1.73$ $\mathrm{cm}$, that is essentially the same values as for $h=3 \mathrm{~cm}$. This corresponds to the critical value $\mu_{1}=1 / 4$ of the parameter $\mu_{1}$ defined in (8). This is due to the fact that the capillary length scale $l=T / \rho c^{2}$ is equal to $0.14 \mathrm{~cm}$ at the $(i q)^{2}$ resonance, which is much smaller than $3 \mathrm{~cm}$.

Experiments have been performed by Longuet-Higgins and Zhang 126, 86, in deep water near the $(i q)^{2}$ resonance. They show good agreement with the theory developed below in section 4 .

Cases in the frame of system (11,6).

Several experiments on waves in an ice plate are reported in the book by Squire et al 106.

i) For the experiments of Takizawa [115], which took place in Lake Saroma in Hokkaido, Japan, the water depth is $h=6.8 \mathrm{~m}$, and other parameters are $E=5.1 \times 10^{8} \mathrm{~N} / \mathrm{m}^{2}, \nu=1 / 3, h_{i}=0.17 \mathrm{~m}$. Then $\lambda / D$ is fixed. The speed $c$ was in the range $[0-9] \mathrm{m} / \mathrm{s}$. This range includes the speed $c=6.09 \mathrm{~m} / \mathrm{s}$, which corresponds to the occurence of the $(i q)^{2}$ resonance (point on the curve $\Gamma$ in figure 6) $(k=2.27, \lambda=1.8, D=0.02)$, as well as the speed $c=8.2 . \mathrm{m} / \mathrm{s}$, which corresponds to the occurence of generalized solitary waves (point on the line $\Delta$ in figure $6(\lambda=1, D \sim 0.01)$. The wavelength of the $(i q)^{2}$ resonance is $18.8 \mathrm{~m}$.

ii) For the experiments of Squire et al [107], which took place in McMurdo Sound in Antarctica, the average water depth is $350 \mathrm{~m}$. The other parameters are $E=4.2 \times 10^{9} \mathrm{~N} / \mathrm{m}^{2}, \nu=0.3, h_{i}=1.6 \mathrm{~m}$. The speed $c$ was in the range $[0-28] \mathrm{m} / \mathrm{s}$. This range includes the speed $c=18.5 \mathrm{~m} / \mathrm{s}$, which corresponds to the occurence of the $(i q)^{2}$ resonance (point on the curve $\Gamma$ in figure 6$)(k=0.038$, $\left.\lambda \sim 10, D \sim 10^{-4}\right)$. The wavelength of the $(i q)^{2}$ resonance is $165 \mathrm{~m}$. The observations of Takizawa [115] and Squire et al 107] are in good agreement with the theoretical results developed in section 4 .

Case in the frame of (1, (7).

A lot of experiments have been performed in the configuration of two superposed fluids. In the experiments of Michallet and Barthélemy [90], the fluids are water and petrol. The density ratio is $\rho=0.78$. The total depth is $10 \mathrm{~cm}$ and the thickness ratio $e=h_{1} / h_{2}$ varies between 0.25 and 10 . Depending on the initial conditions, both types of solitary waves (see figure 7 ) (the 'fast' one bifurcating along $\lambda=\lambda_{0}^{-}$, and the 'slow' one bifurcating along $\lambda=\lambda_{0}^{+}$) can be observed experimentally. Taking $e=0.25$ gives $\lambda_{0}^{+}=21.9$ (that is $c=18.9$ $\mathrm{cm} / \mathrm{s}$ ) and $\lambda_{0}^{-}=0.83$ (that is $c=97.2 \mathrm{~cm} / \mathrm{s}$ ). Taking $e=10$ gives $\lambda_{0}^{+}=4.9$ (that is $c=13.4 \mathrm{~cm} / \mathrm{s}$ ) and $\lambda_{0}^{-}=0.093$ (that is $c=98.1 \mathrm{~cm} / \mathrm{s}$ ). In the case $\lambda=\lambda_{0}^{+}$, there is an additional imaginary eigenvalue $i k$. For $e=0.25, k=21.9$, that is a wavelength of $2.3 \mathrm{~cm}$. For $e=10, k=4.9$, that is a wavelength of 1.2 $\mathrm{cm}$. Note that although Michallet and Barthélemy devoted their experiments to the 'slow' waves, they did not observe the influence of the extra imaginary eigenvalue (see Dias and Il'ichev 33] for a discussion). Recent experiments by Mehta and Sutherland 89] in a three-layer configuration clearly show the presence of generalized solitary waves.

Case in the frame of $\left.(1,17)_{\infty}\right)$. 
The obvious example is the open ocean with a layer of warm water above the cold one. Take for example $\rho=0.998$ for the density ratio and $h_{2}=$ $100 \mathrm{~m}$ for the thickness of the upper (warm) layer. Then a critical value of $\lambda$ is $1 /(1-\rho)=500$, which corresponds to $c=1.4 \mathrm{~m} / \mathrm{s}$, and the wave length of the ripples (corresponding to the pair of eigenvalues $\pm i k$, with $k=\lambda$ ) is $1.3 \mathrm{~m}$. As far as we know, generalized solitary waves have not been observed in the open ocean. However in the course of their investigation of internal-wave disturbances, generated by stratified flow over a sill, Farmer and Smith 41 observed waves resulting from the interaction between a long "internal" mode and a short "external" mode with the same phase speed.

\subsection{Center manifold reduction (finite depth case)}

After having discussed the physical relevance of the waves studied in this paper, we now focus on the reduction procedure. For all these problems one can obtain an estimate on the resolvent operator, of the form

$$
\|\left.\left(i k \mathbb{I}-L_{\mu}\right)^{-1}\right|_{\mathcal{L}(\mathbb{H})} \leq c /|k|
$$

for large $|k|, k \in \mathbb{R}$, where $\mathcal{L}(\mathbb{H})$ is the space of bounded linear operators in $\mathbb{H}$ (see for example [64, 58). It is fortunate that in all these problems the resolvent $\left(i k \mathbb{I}-L_{\mu}\right)^{-1}$ can be obtained quasi-explicitly, especially in the problem (11., (]), because the "bad" trace term in the first component of $L_{\mu}$ makes it difficult to obtain an estimate such as (17) with a choice of basic space other than the space $\mathbb{H}$ we chose. This estimate appears to be essential in our method of reduction to a (small dimensional) center manifold.

For the study of the nonlinear problem (11) the idea is now to use, for the finite depth case, a center manifold reduction which leads to an ordinary differential equation of small dimension. Let us assume that, for values of the (multi)parameter $\mu$ near $\mu_{0}$, the eigenvalues of $L_{\mu}$ lie either in a small vertical strip centered on the imaginary axis, of width tending towards 0 for $\mu \rightarrow \mu_{0}$, or at a distance of order 1 from the imaginary axis. Then the estimate (17) allows us to find a center manifold (see [73], [92], 119], 76]). Indeed, the nonlinear part $N(\mu, \cdot)$ of the vector field $F(\mu, \cdot)$ maps analytically $\mathbb{D}$ into $\mathbb{D}$ for the vector fields (2) and (4). Hence, in such cases, the simple version of the result proved in 119 applies. For the vector field (7), the operator $N(\mu, \cdot)$ maps analytically $\mathbb{D}$ into $\mathbb{H}$, which is not a Hilbert space (however still a Banach space (which is not reflexive)) and is less "regular" than $\mathbb{D}$; so in this case we need the improved result of $\left[76\right.$ where it is needed to replace for instance the space $\mathcal{C}^{0}(\mathbb{D})$ by $\mathcal{C}^{0,1 / 2}(\mathbb{D})$, i.e. continuity in space $\mathbb{D}$ is replaced by Hölder continuity (exponent $1 / 2)$ in this space.

Roughly speaking, all "small" bounded continuous solutions taking values in $\mathbb{D}$ of the system (1) , for values of the (multi)parameter $\mu$ near $\mu_{0}$, lie on an invariant manifold $\mathcal{M}_{\mu}$ which is smooth (however we loose the $C^{\infty}$ regularity) and which exists in a neighborhood of 0 independent of $\mu$. The dimension of 
$\mathcal{M}_{\mu}$ is equal to the sum of dimensions of invariant subspaces belonging to pure imaginary eigenvalues for the critical value $\mu_{0}$ of the (multi)parameter. In other words, the modes corresponding to eigenvalues far from the imaginary axis are functions ("slaves") of the modes belonging to eigenvalues near or on the imaginary axis. In addition, the reversibility property leads to a manifold which is invariant under the reversibility symmetry $S$. The trace of the system (11) on $\mathcal{M}_{\mu}$ is also reversible under the restriction $S_{0}$ of the symmetry $S$.

At this point we should emphasize that the physical relevance of this reduction process is linked with the distance of the rest of eigenvalues to the imaginary axis. So, this validity is going to 0 when the thickness of the bottom layer increases, and in such a case we have to think of another technique.

\section{Finite depth case via reversible normal forms}

The aim of this section is to present a systematic method of study, valid in cases with finite depth layers: for instance we may use this method for solving cases with more than two superposed layers, with or without surface and interfacial tension. We consider the finite depth cases (2), (4), (6), (7), in studying the reduced ODE which gives all solutions staying near 0 . These solutions lie on a low dimensional center manifold, and this ODE is still reversible and its linear part contains the "critical" eigenvalues. Then, we use the normal form technique (see for instance the book [62], especially for reversible normal forms) to simplify the form of the leading orders of the Taylor expansion of the reduced vector field. We shall use a terminology of resonances due to Arnold [1] for describing the form of the reduced linearized operator which corresponds to the eigenvalues lying on the imaginary axis, at the critical value of the parameter.

\subsection{Case of one layer without surface tension}

Let us consider the system (1, 2). When $\lambda<1$, and $\lambda$ not close to 1 , the center manifold is one-dimensional, and the only "small" solutions near 0 belong to the one-parameter family of constant solutions $U=\left(\beta_{0}, 0, \beta_{0}\right)^{t}=\beta_{0} \xi_{0}$, where $\xi_{0}$ is the eigenvector belonging to the eigenvalue 0 of the linear operator $L_{\mu}$.

When $\lambda>1, \lambda$ not close to 1 , the center manifold is 3 -dimensional. A one-parameter family of periodic waves bifurcates from every constant solution. Along this branch, the amplitude increases, starting with amplitude 0 (the constant solution), and the wave length depends regularly on the square of the amplitude. This is a result analogous to the one given by the Lyapunov-Devaney theorem (see 87 for Hamiltonian systems) for finite dimensional reversible systems, despite the occurence of the 0 eigenvalue. The analysis below gives all "small" solutions for $\lambda$ close to 1 , with some details. In particular, for $\lambda \gtrsim 1$, we still obtain the above family of periodic solutions. The first rigorous results on periodic solutions, the so-called Stokes waves (1847), are due to Levi-Civita 81 and Nekrasov [94], and for the solitary wave (for $\lambda<1$ ), they are due to Lavrentiev [80], Friedrichs and Hyers [43], Beale [14]. Below we leave free the Bernoulli 
constant, which allows a better understanding of the family of solutions.

The linearized operator obtained for $\lambda=1$ is denoted by $L_{\mu_{0}}$. Recall that 0 is a triple eigenvalue and there is no other eigenvalue on the imaginary axis.

Let us denote by $\xi_{0}, \xi_{1}, \xi_{2}$ the vectors in $\mathbb{D}$ such that

$$
\begin{aligned}
L_{\mu_{0}} \xi_{0} & =0, L_{\mu_{0}} \xi_{1}=\xi_{0}, L_{\mu_{0}} \xi_{2}=\xi_{1}, \\
S \xi_{0} & =\xi_{0}, S \xi_{1}=-\xi_{1}, S \xi_{2}=\xi_{2}
\end{aligned}
$$

we have

$$
\xi_{0}=(1,0,1)^{t}, \quad \xi_{1}=(0,-(1+y), 0)^{t}, \quad \xi_{2}=\left(0,0,-y-y^{2} / 2\right)^{t} .
$$

In the terminology of Arnold [1], this is a $0^{3+}$ resonance. The 3 -dimensional center manifold is denoted as follows:

$$
U=A \xi_{0}+B \xi_{1}+C \xi_{2}+\Psi_{\nu}(A, B, C),
$$

where $\left.\Psi_{\nu}\right|_{\nu=0}$ is at least of second order in $(A, B, C)$ and $\Psi_{\nu}$ vanishes at 0 . Here $\nu(\mu)$ is a scalar regular function of the parameter defined below, with the help of the normal form, such that $\nu=0$ for $\lambda=1$.

It then results from normal form theory (see for instance 62 p.25 and p.31) that we can choose the coordinates $A, B, C$ by finding a suitable form for $\Psi_{\nu}$ up to a certain order, such that for any fixed $p$, the system reads

$$
\begin{aligned}
& \frac{d A}{d x}=B, \\
& \frac{d B}{d x}=C+A \phi_{\nu}\left(A, B^{2}-2 A C\right)+R_{B}\left(A, B^{2}, C, \nu\right), \\
& \frac{d C}{d x}=B \phi_{\nu}\left(A, B^{2}-2 A C\right)+B R_{C}\left(A, B^{2}, C, \nu\right),
\end{aligned}
$$

where $\phi_{\nu}$ is a polynomial in its arguments, of degree $p$ in $(A, B, C)$, and $R_{B}$, $R_{C}$ are even in $B$, due to reversibility (the vector field anticommutes with

$$
(A, B, C) \mapsto(A,-B, C) .
$$

Moreover

$$
\left|R_{B}\right|+\left|B R_{C}\right|=O\left\{(|A|+|B|+|C|)^{p+1}\right\}
$$

holds. We may compute the principal part of the polynomial $\phi_{\nu}$ (see for instance a similar computation in the appendix of 61):

$$
\begin{aligned}
\phi_{\nu}\left(A, B^{2}-2 A C\right) & =\nu+a A+b\left(B^{2}-2 A C\right)+c A^{2}+\cdots \\
\nu & =\frac{3}{2}(1-\lambda)+O\left[(1-\lambda)^{2}\right], \\
a & =3[1+O(\nu)] .
\end{aligned}
$$

In $\phi_{\nu}$ we consider all coefficients $(a, b, c, \ldots)$ as functions of $\nu$ instead of $\lambda$, for a better comfort. 
A nice property of $(18,19,20)$ is that, if the higher order terms $R_{B}, R_{C}$ which are not in normal form are suppressed, then the "truncated" system is integrable. Indeed, we have the two first integrals

$$
\begin{aligned}
B^{2}-2 A C & =K, \\
C-\Phi(A, K, \nu) & =H,
\end{aligned}
$$

where

$$
\Phi(A, K, \nu)=\int_{0}^{A} \phi_{\nu}(s, K) d s .
$$

For $(H, K)$ fixed, all trajectories in the $(A, B, C)$ space are given by

$$
\begin{aligned}
B^{2} & =f_{H, K}(A), \\
C & =H+\Phi(A, K, \nu),
\end{aligned}
$$

where

$$
f_{H, K}(A) \stackrel{\text { def }}{=} K+2 H A+2 A \Phi(A, K, \nu)
$$

The corresponding curves $B^{2}=f_{H, K}(A)$ are deduced from figure 8, depending on the sign of $\nu$ and on the values of the first integrals $(H, K)$. In all cases we have a family of equilibria implicitly given by

$$
B=0, \quad C+A \phi_{\nu}(A,-2 A C)=0,\left(\text { i.e. } \partial_{A} f_{H, K}(A)=0\right),
$$

which correspond to the curves in the $(H, K)$ plane. These equilibria may be
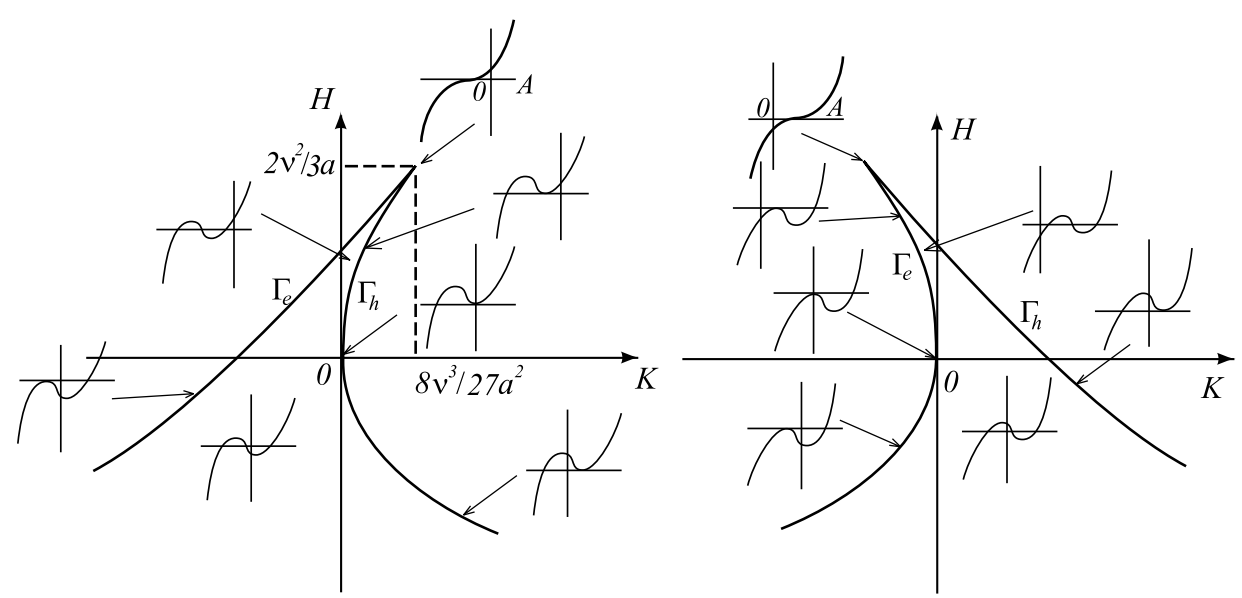

Figure 8: Different graphs of $A \mapsto f_{H, K}(A)$ for $\nu>0$ (left), and $\nu<0$ (right)

elliptic or hyperbolic depending on the branch $\Gamma_{e}$ or $\Gamma_{h}$ where $(H, K)$ is sitting. On the branch on the right, for $\nu>0$ and $H=K=0$, there is one solution homoclinic to 0 . On the branches denoted by $\Gamma_{h}$ in the $(H, K)$ planes for $\nu>0$ 
or $\nu<0$, the equilibria are limit points of homoclinics corresponding to solitary waves. Other small bounded solutions are periodic (cnoidal waves), corresponding to the positive part of $f_{H, K}$ when the curve intersects transversally the axis $B=0$.

We can check that the solitary waves are of elevation: for $\nu>0, H=K=0$, we have indeed for the principal part

$$
A(x) \sim-\frac{2 \nu}{3 \cosh ^{2}(\sqrt{\nu / 2} x)},
$$

hence

$$
\begin{aligned}
& \eta=Z(x) \sim-\int_{-1}^{0} \beta(x, y) d y \sim-A(x)>0, \\
& x \sim \xi, \quad \lambda=1-\nu .
\end{aligned}
$$

Now, we need to prove that what is true on the normal form of the reduced vector field is still true for the full vector field. In particular the curve of symmetric equilibria persists (by implicit function theorem) and the two non zero eigenvalues of the linearized operator are either purely imaginary (elliptic case), or real symmetric (hyperbolic case). There is one equilibrium for which 0 is a triple eigenvalue. There is a homoclinic connection to every hyperbolic equilibrium, as may be shown by taking the one-dimensional unstable manifold of each of these hyperbolic equilibria, and showing that this curve intersects transversally the plane of symmetric points $B=0$, as for the normal form. Hence the trajectory obtained by completing in a symmetric way is an homoclinic curve. A complete proof of such a persistence in the three dimensional phase space may be found for instance in [61]. We can sum up these results by the following theorem:

Theorem 1 Assume that a 3-dimensional reversible vector field having a fixed point at 0 has a $0^{3+}$ resonance. Then the phase portrait near 0 , of the vector field for a fixed value (near 0) of the bifurcation parameter, is generically the same as for the normal form (18, 19,20) truncated at quadratic order.

Corollary 2 The above theorem applies for describing the travelling wave solutions in the space $\mathbb{D}$ of the water wave problem 2.1.1 (finite depth, no surface tension) near the equilibrium, for $\lambda$ near 1 .

Remark: Note that $U=0$ corresponds here to a uniform flow of velocity $c$, and that other constant solutions near 0 correspond in fact to uniform flows moving at a velocity slightly different than $c$. Since $c$ was chosen arbitrarily as the velocity scale, solitary waves corresponding to solutions homoclinic to nonzero constant solutions are in fact homoclinic solutions to 0 , with the right choice of the velocity scale. Therefore, there is a unique form of solitary waves (see figure 9), due to the arbitrariness of the definition of the parameter $\lambda$ (with an arbitrary $c$ !). 


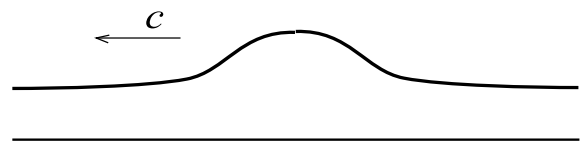

Figure 9: Shape of the solitary wave in the case of one layer without surface tension.

\subsection{Case of one layer with surface tension}

Let us consider the system (1, , (1). The eigenvalues of the linear operator $L_{\mu}$ (for $c_{1}=0$ ), are given by $\sigma=i k$, with $k$ satisfying (12), and figure 5 gives the position of the four closest eigenvalues to the imaginary axis. For the study of solutions of (11) near 0 , there are three main "interesting" cases to be considered:

(i) $(b, \lambda)$ is near $\Delta^{+}=\{\lambda=1, b>1 / 3\}$; in this case, $L_{\mu_{0}}$ has only a double 0 eigenvalue on the imaginary axis $\left(0^{2+}\right.$ resonance),

(ii) $(b, \lambda)$ is near $\Delta^{-}=\{\lambda=1,0<b<1 / 3\}$; in this case, $L_{\mu_{0}}$ has a double 0 eigenvalue, and a pair of simple imaginary eigenvalues $\pm i q$ on the imaginary axis $\left(0^{2+}(i q)\right.$ resonance $)$,

(iii) $(b, \lambda)$ is near $\Gamma$ (left part); in this case $L_{\mu_{0}}$ has only a pair of double imaginary eigenvalues $\pm i q$ on the imaginary axis $\left((i q)^{2}\right.$ resonance).

The system (1.,6) leads to cases (ii) and (iii) as well, and can be treated similarly. When $(b, \lambda)$ is close to $(1 / 3,1)$ a specific study is needed, because at this point the eigenvalue 0 is quadruple (see [56] for this case). There are other interesting cases, for instance when one has two pairs of resonating eigenvalues on the imaginary axis (the $(i q)(2 i q)$ resonance is the most special because of the occurence of heteroclinics between periodic solutions, see [13]). However we shall not detail their study here, since it is always in the same spirit, and we restrict our presentation to the most typical cases.

\subsubsection{Case (i): $0^{2+}$ resonance}

This case was first solved by Amick and Kirchgässner [4]. It is also studied in particular in the papers [74, 101, 64]. The method used in [64] is the one we present here (however without fixing the parameter $c_{1}=0$ ). Here the center manifold is two dimensional. Let us define by $(A, B)$ the (real) coordinates (or "amplitudes"), associated with the choice of eigenvectors

$$
\xi_{0}=(0,0,1)^{t}, \xi_{1}=(-1,-(y+1), 0)^{t} .
$$

Then, we need to know how the reversiblity symmetry $S_{0}$ acts on $(A, B)$. There are two theoretical possibilities: $(A, B) \rightarrow(A,-B)$ or $(-A, B)$. Here, as in all water-wave problems, the first case holds. This is the $0^{2+}$ resonance. Then the normal form (see for instance [62]), truncated at leading orders, reads

$$
\left\{\begin{array}{l}
\frac{d A}{d x}=B \\
\frac{d B}{d x}=\nu A+a A^{2}+\delta c_{1},
\end{array}\right.
$$


where one can compute explicitly the coefficients (see for instance [64]), as functions of the parameters:

$$
\begin{aligned}
& \nu=(b-1 / 3)^{-1}(\lambda-1)+O\left(\left|c_{1}\right|+|\lambda-1|^{2}\right), \\
& a=-3 / 2(b-1 / 3)^{-1}\left[1+O\left(|\lambda-1|+\left|c_{1}\right|\right)\right], \\
& \delta=(b-1 / 3)^{-1} b\left[1+O\left(|\lambda-1|+\left|c_{1}\right|\right)\right] .
\end{aligned}
$$

We notice the blowing up of the coefficients when $b$ tends towards $1 / 3$, due to the change of dimension of the central system at this point (it becomes 4dimensional). Here, the two conjugate equilibria (both corresponding to a flat free surface) are denoted by $A^{-}<A^{+}$. They exist provided that $c_{1}>-\frac{(\lambda-1)^{2}}{6 b}$. The equilibrium $A^{-}$is hyperbolic while the equilibrium $A^{+}$is elliptic. The vector field (24) is integrable, and its phase portrait is given in figure 10. For any fixed $c_{1}$, there is a one parameter family of periodic solutions, and a solution homoclinic to the hyperbolic equilibrium. All these solutions disappear after the saddle-node bifurcation when $c_{1}<-\frac{(\lambda-1)^{2}}{6 b}$. For $c_{1}=0$ and $\lambda>1$, the homoclinic solution of the truncated system is given by

$$
A(x)=\frac{\lambda-1}{\cosh ^{2}\left(\nu^{1 / 2} x / 2\right)}
$$

Because our system is two-dimensional and reversible, it is easy to show that these phase portraits fully persist for the complete system. We sum up these results in the following theorem:

Theorem 3 Assume that a two-dimensional reversible vector field has a fixed point at the origin for the parameter value 0 , and assume that it has a $0^{2+}$ resonance for its linearized part. Then the phase portraits near 0 of the vector field, for fixed values of the parameter $\left(\left(\nu, c_{1}\right)\right.$ here $)$ near 0 , are generically the same as for the normal form (24).

Corollary 4 The above theorem applies for describing travelling waves solutions of the water wave problem 2.1.2 (finite depth, large surface tension such that $b>1 / 3)$ for $\lambda$ near 1 .

We observe that $A(x)>A^{-}$, hence the homoclinic solution corresponds to a "solitary wave" of depression (see figure 11) for the problem (1, 1), whose principal part follows directly from (25).

Remark: Fixing $c_{1}=0$ leads to an artificial distinction between the cases $\lambda>1$ and $\lambda<1$, since this is just a matter of choosing the suitable constant flow for the velocity scaling (the one which is an hyperbolic equilibrium).

\subsubsection{Case (ii): $0^{2+}(i q)$ resonance}

This case was treated in the spirit of this review in the work [64]. Here the center manifold is four dimensional. Let us denote by $\pm i q$ the pair of simple eigenvalues depending on $b$, such that $q=\left(1+b q^{2}\right) \tanh q$, and define by $(A, B)$ the (real) 


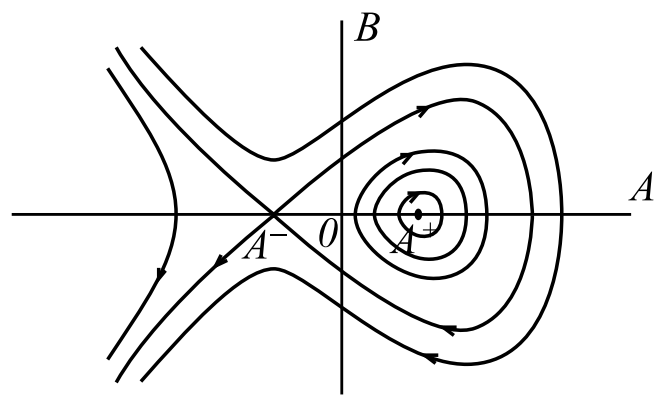

Figure 10: Phase portrait of the 2D vector field (24) [case(i)], for $c_{1}>-\frac{(\lambda-1)^{2}}{6 b}$.

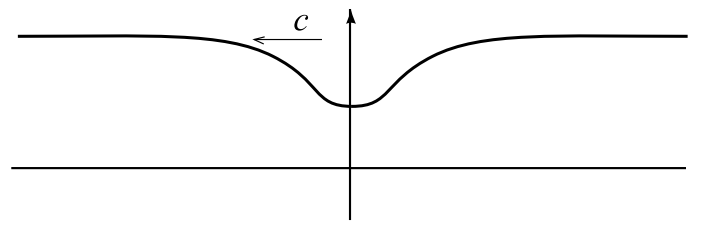

Figure 11: Shape of the solitary wave of depression in the case of one layer with strong surface tension

amplitudes and $C$ the complex one, corresponding to the oscillating mode. Then the reversiblity symmetry $S_{0}$ acts on $(A, B, C, \bar{C})$ as follows: $(A, B, C, \bar{C}) \rightarrow$ $(A,-B, \bar{C}, C)$. This is a $0^{2+}(i q)$ resonance. The normal form, truncated at quadratic order, reads

$$
\left\{\begin{array}{l}
\frac{d A}{d x}=B \\
\frac{d B}{d x}=\nu A+a A^{2}+c|C|^{2}+\delta c_{1}, \\
\frac{d C}{d x}=i C\left(q+\nu_{1}+d A\right),
\end{array}\right.
$$

where the (real) coefficients $\nu, a, \delta$ are the same as for case (i), and $c, \nu_{1}, d$ may also be explicitly computed in terms of the parameters $\left(\lambda, b, c_{1}\right)$ (see 64 where they are computed for $\left.c_{1}=0\right)$. We notice that $a>0$ and we have

$$
\begin{aligned}
\nu_{1} & =O\left(|\lambda-1|+\left|c_{1}\right|\right), \quad d=O(1), \\
c & =(1 / 3-b)^{-1}\left(1+\frac{\sinh 2 q}{q}+\text { h.o.t. }\right)>0
\end{aligned}
$$

where $\left(\lambda-1, c_{1}\right)$ is close to 0 . This system is indeed integrable, with the two first integrals

$$
\begin{aligned}
& H=|C|^{2}, \\
& K=B^{2}-(2 / 3) a A^{3}-\nu A^{2}-2\left(c H+\delta c_{1}\right) A .
\end{aligned}
$$



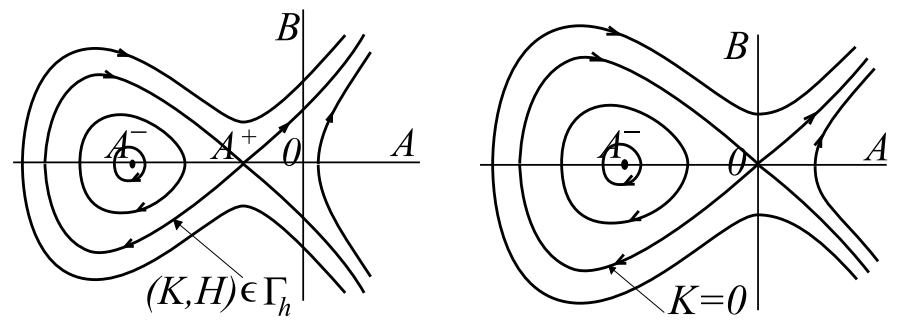

Figure 12: Phase portraits in the $(A, B)$ plane of the vector field $(26)$ for $c_{1}=$ $0, \nu>0 \quad(\lambda<1)$; left side: $0<c H<\nu^{2} / 4 a$, right side: $H=0$.

Fixing $c_{1}=0$ to simplify the discussion, we see again on figure 8 (after an obvious scaling) the various graphs of the functions $f_{H, K}(A)=(2 / 3) a A^{3}+$ $\nu A^{2}+2 c H A+K$ depending on $(K, H)$, for $\nu>0$ (left), and for $\nu<0$ (right) ( $\nu$ has the sign opposite of $\lambda-1$, since $b<1 / 3$ ).

In this case, for the normal form vector field, the curves $\Gamma_{h}$ and $\Gamma_{e}$ in the $(K, H)$ plane correspond to families of periodic solutions, where the $C$ component is not 0 , except for $H=0$, where this gives the conjugate constant flow (as above). Now, we have other types of periodic solutions and quasi-periodic solutions corresponding to the interior of the triangular region in $(K, H)$ plane. The curves $\Gamma_{h}$ correspond to the existence of homoclinic solutions, one homoclinic to 0 for $\lambda<1(\nu>0, H=K=0)$, and all others homoclinic to some periodic solution. Figure 12 gives in the $(A, B)$ plane the phase portrait of all small bounded solutions (left side) for $0<c H<\nu^{2} / 4 a, c_{1}=0$, and for $H=0$ (right side). Notice that the homoclinic solution to $A_{+}$corresponds here to a generalized solitary wave for the problem (1, (1), tending at infinity towards a periodic wave. Note that $A_{+} \sim-c H / \nu$ when $|H|$ is very small, meaning that oscillations at infinity are then very small in this case. For $H=0$ this corresponds to a solitary wave of elevation for the problem (1, 他. For $\lambda>1$ we have analogous phase portraits where, for instance for $H=K=0$, we have a solution homoclinic to $A_{+} \neq 0$. This limit equilibrium corresponds to the flow conjugate to 0 , and might be chosen a priori as the origin (instead of the previous origin) if we change the scale $c$ for velocities (see the discussion for case (i)). Then $\lambda$ would become $\lambda^{\prime}$ with the new scaling, and $\lambda>1$ would become $\lambda^{\prime}<1$.

The natural mathematical problem consists now in proving persistence results when considering the full system, not only reduced to its normal form. In summary, the persistence of periodic solutions of the normal form can in general be performed, through an adaptation of the Lyapunov-Schmidt technique [64, 82]. The persistence of quasi-periodic solutions is much more delicate, and can only be performed in a subset of the 2 dimensional space of first integrals, where these solutions exist for the normal form. For a fixed value of the bifurcation parameter $\nu$, quasi-periodic solutions of the perturbed reversible vector field exist for $(H, K)$ lying in a region which is locally the product of a line by a 
Cantor set (see [64]). The persistence of pairs of reversible solutions (invariant under the reversibility symmetry) homoclinic to periodic solutions, provided that they are not too small, is proved for instance in [109] and [64] (see figure 13). For the normal form, there is a family of orbits homoclinic to a family of periodic solutions whose amplitude can be chosen arbitrarily small. Such a case (ii) has been investigated by many authors (see for instance [15], [113] and [82]). There are homoclinic solutions to oscillations at infinity whose size is smaller than any power of the bifurcation parameter, corresponding to the fact that we cannot avoid such oscillations when we consider the full untruncated system. The extremely delicate aspect of exponentially small and still existing oscillations was proved by Sun and Shen [113 on the water wave problem (1, 1 , , and is thoroughly studied by Lombardi for a wide class of problems (including the water-wave problem) in 82. Moreover, despite the fact that a solution homoclinic to 0 exists for the normal form (26), this is not true in general for the full system (see [83]), even though one can compute an asymptotic expansion up to any order of such an homoclinic (non existing) "solution"! (see 84 for an extensive study of the phenomenon). The difficulty comes from the fact that there is only one unstable direction and one symmetric stable direction for the origin $(\nu>0)$. Indeed, the two-dimensional unstable manifold of a periodic orbit near 0 becomes one-dimensional when the amplitude of the oscillation vanishes. In fact, this two-dimensional unstable manifold (identical to the twodimensional stable manifold for the normal form) intersects transversally the two-dimensional subspace invariant under the symmetry reversibility $(B=0$, $C$ real), (2 intersection points) for the 4-dimensional normal form vector field. For a large enough size of the periodic orbit for the perturbed vector field, its unstable manifold is shown to intersect transversally the plane ( $B=0, C$ real), in two points, as for the normal form. This shows the persistence, for the full vector field, of two reversible solutions homoclinic to this closed orbit. Now, it results from 84 that, as soon as the radius of the periodic orbit is smaller than a critical value, there is a loss of transversality for the perturbed vector field, and that the generic minimal size of the limiting oscillation is $O\left(C(l) \exp \left[-l q / \nu^{1 / 2}\right]\right)$, with $l<\pi(l=\pi$ would be the optimal result here, but not yet obtained, see [84]). While the result on non existence of solitary waves is generic here, there is a precise proof that there are no true solitary waves (of elevation here) near $b=1 / 3$ (see $[110$ ), and the result for $b<1 / 3$ (not near $1 / 3$ ) is not known, though a not completely rigorous analysis suggests that there are no such solutions (see 124] and 27]). We summarize these results in the following rough theorem:

Theorem 5 Assume that a 4-dimensional reversible vector field has a fixed point at 0 and has $a 0^{2+}(i q)$ resonance for its linear part. Then, in a neighborhood of 0 , near the critical value of the bifurcation parameter, the small periodic solutions of frequencies close to $q$ are generically given by the normal form (20), and lie in a one-parameter family. There is in addition a two-parameter family of periodic and quasi-periodic solutions. Moreover, each hyperbolic periodic solution of frequency close to $q$ has two reversible homoclinic connections, provided 


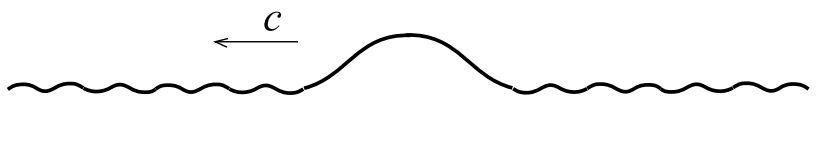

Figure 13: Shape of the generalized solitary waves in case (ii).

that the diameter of this periodic solution is larger than an exponentially small quantity.

More details may be found in 84 .

Corollary 6 The above theorem applies for describing travelling waves of the water wave problem 2.1.2 for $\lambda$ near 1 and $b<1 / 3$ (small surface tension). It also applies to the problem of travelling waves 2.2 under an elastic plate (6) for $\lambda$ near 1 (see the line $\Delta$ in figure 6 and see 9 ]).

\subsubsection{Case (iii): $(i q)^{2}$ resonance}

This case occurs for $(b, \lambda)$ near the curve $\Gamma$ of the parameter plane in figure 5, and was first treated in [63]. Here the center manifold is four dimensional. Let us denote by $\pm i q$ the pair of double eigenvalues at criticality, and define by $(A, B)$ the complex amplitudes corresponding respectively to the eigenmode and to the generalized eigenmode. This case is often denoted by "1:1 reversible resonance". We can always assume that the reversibility symmetry $S_{0}$ acts as $(A, B) \rightarrow(\bar{A},-\bar{B})$. The normal form (see for instance [62]) reads at any order (making $c_{1}=0$, which does not restrict the study, since $\lambda$ is not close to 1 ):

$$
\begin{aligned}
& \frac{d A}{d x}=i q A+B+i A P\left[\nu,|A|^{2}, i / 2(A \bar{B}-\bar{A} B)\right] \\
& \frac{d B}{d x}=i q B+i B P\left[\nu,|A|^{2}, i / 2(A \bar{B}-\bar{A} B)\right]+A Q\left[\nu,|A|^{2}, i / 2(A \bar{B}-\bar{A} B)\right],
\end{aligned}
$$

where $P$ and $Q$ are real polynomial of degree one in their arguments, for the cubic normal form. Let us define more precisely the coefficients of $Q$ :

$$
Q(\nu, u, v)=\nu+q_{2} u+q_{3} v,
$$

which means that for $\nu>0$ the eigenvalues are at a distance $\sqrt{\nu}$ from the imaginary axis, while, for $\nu<0$, they sit on the imaginary axis. Values $\nu>0$ correspond to points in the plane $(b, \lambda)$ above the curve $\Gamma$, and $\nu$ is of the order of the distance to this curve (the precise expression of $\nu$ in terms of the parameters is given in [34). The vector field (28) is integrable, with the two following first 

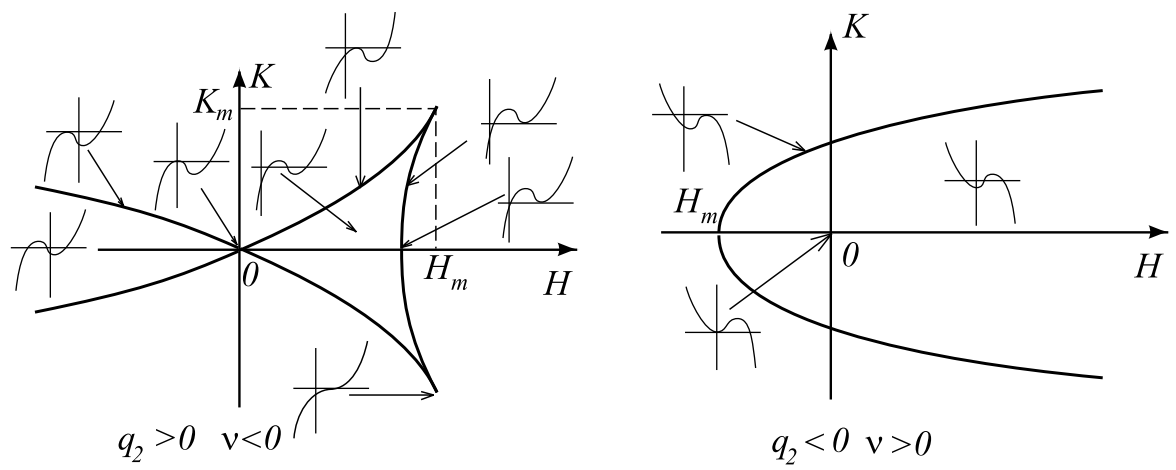

Figure 14: Different graphs of $u \mapsto f_{K, H}(u)$ depending on the parameters $H$ and $K$

integrals:

$$
\begin{aligned}
& K=i / 2(A \bar{B}-\bar{A} B), \\
& H=|B|^{2}-\int_{0}^{|A|^{2}} Q(\nu, u, K) d u .
\end{aligned}
$$

It is then possible to describe all small bounded solutions of (28). Indeed, we obtain

$$
\left(\frac{d|A|^{2}}{d x}\right)^{2}=f_{K, H}\left(|A|^{2}\right)
$$

where

$$
f_{K, H}\left(|A|^{2}\right)=2 q_{2}|A|^{6}+4\left(\nu+q_{3} K\right)|A|^{4}+4 H|A|^{2}-4 K^{2} .
$$

We show in figure 14 various graphs for the functions $f_{K, H}$ depending on $(K, H)$, for $\nu>0, q_{2}<0$ (right), and for $\nu<0 q_{2}>0$ (left), which are the most interesting cases. The change $\left(q_{2}, H, u\right) \mapsto\left(-q_{2},-H,-u\right)$ leaves $f_{K, H}(u)$ unchanged. It results that the relevant graph (we need $|A|^{2}>0$ ) of $f_{K, H}$ for $\nu<0$, and $q_{2}>0$ corresponds to the side $H>0$ of the left part of figure 14, while for $\nu<0, q_{2}<0$ we need to consider the side $H<0$ of the left part of figure 14. Notice that for $q_{2}>0, \nu>0$ there is no small bounded solution other than 0 .

Looking at these graphs, where in particular any tangency to the $u$ axis, on the positive side, corresponds to a periodic solution of frequency close to $q$, it is clear that we obtain for a fixed $\nu$, two-parameter families of periodic and quasiperiodic solutions and, for $q_{2}<0, \nu>0$ a circle of solutions homoclinic to 0 with exponentially damped oscillations at infinity, while for $q_{2}>0, \nu<0$ we have a one-parameter family of circles of solutions homoclinic to periodic solutions 


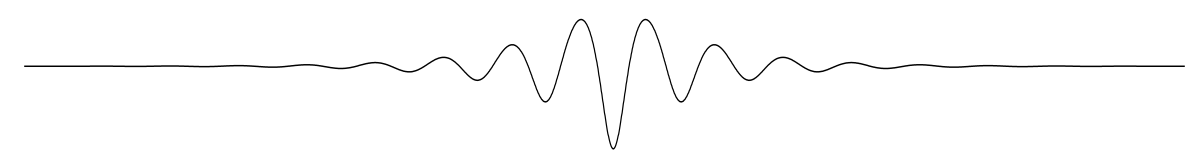

Figure 15: Depression wave for case (iii)

(as in case (ii)) where the amplitude is minimum at $x=0$. The computation of the coefficients of the normal form (28) corresponding to the system (4) is performed by Dias \& Iooss in [34]; it is shown that $q_{2}<0$ holds all along $\Gamma$. For the ice problem (6), the present case (iii) occurs along the curve $\Gamma$ of figure 6, and the coefficient $q_{2}$ can have either sign (see 97]), depending on the water depth. The mathematical problem of persistence of the above solutions of the normal form system, for the full vector field, is done in an analogous way as for case (ii). This means in particular that we have a one-parameter family of pairs of reversible homoclinics to periodic orbits. For the homoclinic to 0, it is in fact simpler than case (ii). This is due to the fact that the unstable manifold of 0 (identical to the stable manifold, for the normal form) is twodimensional, and intersects transversally in two points the plane of symmetric points ( $A$ real, $B$ pure imaginary). It gives the persistence of two reversible homoclinic orbits, corresponding to two different "bright" solitary waves, with exponentially damped oscillations at infinity: one has a crest in the middle (elevation wave), and the other has a through in the middle (depression wave) (see figure 15 for the depression wave). Note that this type of solutions has been experimentally observed [86, 126], 107], 115] at least when some forcing is present. The forcing can be an obstacle at the bottom, wind on the surface, a moving load on the surface in the case of ice experiments.

The complete proofs on persistence for periodic and homoclinics can be found in 68], and for quasi-periodic solutions, it is shown in 67] (the method applies directly here with very slight modifications), that persistence holds true in a subset, locally looking like the product of a curve by a Cantor set, of the region of the $(K, H)$ plane where these quasi-periodic solutions exist for the normal form. We sum up these results in a rough theorem:

Theorem 7 Assume that a 4-dimensional reversible vector field has a fixed point at the origin, and has a $(i q)^{2}$ resonance for its linear part. Then, in a neighborhood of 0 , and near the critical value of the bifurcation parameter (possibly only on one side of criticality), there is a one-parameter family of periodic solutions of frequency near $q$ and a two-parameter family of other periodic and quasi-periodic solutions. Moreover, we have generically one of the two cases, depending on the sign of a certain nonlinear coefficient $\left(q_{2}\right.$ in (29)):

case 1: for bifurcation parameter values which lead to four non purely imaginary eigenvalues for the linearized operator, and for $q_{2}<0$, there are two reversible orbits homoclinic to 0 ;

case 2: for bifurcation parameter values which lead to four purely imagi- 
nary eigenvalues for the linearized operator, and for $q_{2}>0$, there are two oneparameter families of reversible orbits homoclinic to the "hyperbolic" periodic solutions of frequencies near $q$.

Corollary 8 The above theorem applies for describing travelling waves of the water wave problem 2.1.2 for $(b, \lambda)$ near the curve $\Gamma$ of figure 5 (case 1) (see [34]). This theorem also applies to the problem of travelling waves under an elastic plate 2.2 for $(D, \lambda)$ near the curve $\Gamma$ of figure 6 (cases 1 and 2) (see [97]).

Remarks: For these results on homoclinics, it should be mentioned that the decay at infinity is exponential. There are degenerate cases (codimension two situations) where this is no longer true. For example when the coefficient $q_{2}$ is close to 0 (see [57]) there exists in general (for $\nu=0$ ) an homoclinic to 0 , with a polynomial decay at infinity. This case may occur in examples having more parameters, such as with several superposed layers. It should be noticed that this phenomenon is in fact different from the similar property of polynomial decay that we shall meet for cases with an infinitely deep layer. Both phenomena are due to different causes.

For problems with several bounded superposed layers, with surface and (or) interfacial tension, there are always values of the parameters where cases (i), (ii), (iii) occur. They can be treated in the same way. More complex bifurcations may occur, for example in case (iii) when $q_{2}$ changes sign. Such a case is a codimension-2 singularity and is partly studied in [35], and completed in [121].

\subsubsection{Bifurcation of plethora of solitary waves}

So far, we have discussed the solitary waves that one can obtain via the normal form technique. In fact this technique provides only a small portion of the existing solitary waves, in particular in cases (ii) and (iii) above. What happens is that it is possible to combine several solitary wave solutions together and still obtain a solution of the problem, a so-called multibump solitary wave (see figure 16 for the profile of such a wave - this type of profile has been obtained numerically in [37] on the full water-wave equations). This can be done as soon as one is sufficiently far from the bifurcation curve. In practice, this distance can be exponentially small. The formation of multibump solutions has been studied in [26], [23], 25], [24] (the first three works deal with a model differential equation), they all use a hamiltonian formulation. We do not intend to describe this process in detail here (it would make the paper much longer!). Rather, we follow the formal approach used in 125], and we concentrate on case (iii). Similar results occur for case (ii).

In the last subsection, we showed that at each order the $(i q)^{2}$ resonance normal form admits two reversible homoclinic solutions provided certain coefficients have the correct sign. The corresponding solutions of the water-wave problem are modulated wavepackets whose envelopes are symmetric and decay exponentially to zero at infinity. In the middle, one wave has a central crest (elevation wave), while the other wave has a central trough (depression wave). As is well known, the normal form (28) yields the nonlinear Schrödinger (NLS) 


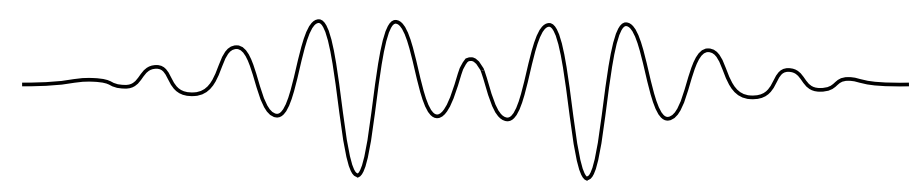

Figure 16: multibump solitary wave resulting from the superposition of two depression waves near the $(i q)^{2}$ resonance

equation to leading order, and of course the NLS equation admits two symmetric envelope-soliton solutions. But one can also construct small-amplitude asymmetric solitary waves, by translating the crests of a symmetric solitary wave relative to its wave envelope. The problem is that such asymmetric waves do not persist when considering the full system. Exponentially small terms come into play! Shifting the carrier oscillations relative to the envelope leads to the appearance of growing oscillations of exponentially small amplitude on one side of the wave packet. However, due to nonlinearity, this growing tail evolves into a new wavepacket and it can be shown that, for certain values of the phase of the carrier oscillations, the whole disturbance terminates, resulting in a solitary wave with two wavepackets. Otherwise, a third wavepacket is generated and the process continues. The main result is that there exists a countable infinity of symmetric and asymmetric multibump solutions. But, unlike the solitary waves obtained in the previous subsection, each of these multibump solitary waves bifurcates at a certain finite amplitude.

When the parameters $b$ and $\lambda$ are close to the critical point $(b, \lambda)=(1 / 3,1)$, which corresponds to the occurence of a quadruple eigenvalue 0 , it can be shown, via center manifold reduction and a normal form argument (see [56]), that the problem essentially reduces to the fourth-order differential equation

$$
y_{x x x x}+P y_{x x}+y-y^{2}=0
$$

where $y$ is directly related to the elevation of the free surface and $x$ to the horizontal coordinate. When the parameter $P$ is equal to 2 , one is along the curve $\Gamma$ in figure 5. When the parameter $P$ is equal to -2 , one is along the dashed line in figure 5. Equation (32) has been studied a lot in 23] and [26]. Using the fact that (32) is a hamiltonian system, these authors proved the existence of an infinity of homoclinic orbits and the presence of spatial chaos. They showed rigorously that (32) admits a unique (up to translations) homoclinic orbit for $P \leq-2$, (i.e. in between the dashed curve and the half line $\Delta$ of figure 5), while for $P$ in $(-2+\varepsilon, 2)$ it has at least two small-amplitude homoclinic orbits. What happens at $P=-2$ is that the unique orbit can bifurcate into a countable infinity of multimodal homoclinic orbits. As $P$ is increased towards 2 (i.e. one goes from the dashed curve towards the curve $\Gamma$ in figure 5 ), the domain of existence of each orbit reaches a limit (turning) point before the value $P=2$, except for one orbit which can be followed all the way towards $P=2$. This orbit is nothing else than the depression wave found earlier (near the half line $\Delta$ )! 
Some of these multimodal homoclinic orbits have been computed numerically for the full water-wave problem in [37]. As said above it was found in [23] that, close to each turning point of a branch of homoclinic, for $P \sim 2$ (i.e. next to the curve $\Gamma$ in our context), there is a bifurcation into a branch of asymmetric homoclinic orbits.

\subsection{Case of two layers without surface tension}

In this case [see system (11,7), and the dispersion relation (14)], 0 is always an at least double eigenvalue of $L_{\mu}$, with the two independent eigenvectors

$$
\begin{aligned}
& \xi_{0}=(1-\lambda, e \lambda, 0,1-\lambda, e \lambda)^{t}, \\
& \xi_{0}^{\prime}=(0,1,0,0,0,1)^{t},
\end{aligned}
$$

which satisfy $S \xi_{0}=\xi_{0}, S \xi_{0}^{\prime}=\xi_{0}^{\prime}$.

When there is no other eigenvalue on the imaginary axis, this just gives near 0 the two-parameter family of stationary solutions of (11.7): $U=\alpha \xi_{0}+\beta \xi_{0}^{\prime}$, corresponding to the freedom on the horizontal velocity in each layer.

When there is a pair of simple eigenvalues on the imaginary axis, in addition to the double 0 eigenvalue, we have near 0 , a family of periodic waves, bifurcating from any of the above equilibria. This situation is similar to system (11,2), for $\lambda>1$ (not close to 1 ), and to system (1, 1 1 ), for $\lambda<1,(b, \lambda)$ not close to $\Delta$.

When $\lambda=\lambda_{0}^{ \pm}(e, \rho), 0$ is a quadruple eigenvalue with two additional (generalized) eigenvectors: $\xi_{1}$ and $\xi_{2}$ such that

$$
\begin{aligned}
& L_{\mu_{0}} \xi_{1}=\xi_{0}, S \xi_{1}=-\xi_{1}, \\
& L_{\mu_{0}} \xi_{2}=\xi_{1}, S \xi_{2}=\xi_{2} .
\end{aligned}
$$

If there is no other eigenvalue on the imaginary axis, which is the case for $\lambda=\lambda_{0}^{-}$ (see figure 7), this is a $00^{3+}$ resonance. This case leads to a four-dimensional center manifold

$$
U=A \xi_{0}+B \xi_{1}+C \xi_{2}+D \xi_{0}^{\prime}+\Psi_{\nu}(A, B, C, D),
$$

where the dynamics is ruled by an ODE of the form (see 62])

$$
\begin{aligned}
& \frac{d A}{d x}=B, \\
& \frac{d B}{d x}=C+A \phi_{\nu}\left(A, B^{2}-2 A C, D\right)+R_{B}\left(A, B^{2}, C, D, \nu\right), \\
& \frac{d C}{d x}=B \phi_{\nu}\left(A, B^{2}-2 A C, D\right)+B R_{C}\left(A, B^{2}, C, D, \nu\right), \\
& \frac{d D}{d x}=B R_{D}\left(A, B^{2}, C, D, \nu\right),
\end{aligned}
$$

where $\phi_{\nu}$ is a polynomial in its arguments, of degree $p$ in $(A, B, C, D)$, and $R_{B}$, $R_{C}, R_{D}$ are even in $B$, due to reversibility. Moreover

$$
\left|R_{B}\right|+\left|B R_{C}\right|+\left|B R_{D}\right|=O\left\{(|A|+|B|+|C|+|D|)^{p+1}\right\}
$$


holds. The normal form here is analogous to the system $(18,19,20)$, except that there is the additional first integral $D$. We recover easily the two-parameter family of reversible equilibria: $B=0, C=C_{0}(A, D)$, where $A$ and $D$ are arbitrary. The study of the normal form is the same as for $(18,19,20$ ) (see figure 8), after a small change in the parameter $\nu$ (at leading orders). The persistence of the phase portraits in the four-dimensional space, for the full vector field, may be proved with the same argument as in $\S 4.1$, because the linear subspace of symmetric points is now three-dimensional $(B=0)$. For example, periodic and homoclinic orbits cross transversally the space $B=0$ for the normal form. Then it can be proved that they persist for the full vector field.

Now, we have the other possible situation $\lambda=\lambda_{0}^{+}$(see figure 17) which is analogous to case (ii) of $\S 4.2$, with a pair of simple eigenvalues on the imaginary axis, in addition to the quadruple eigenvalue at 0 . This is a $00^{3+}(i q)$ resonance. The values of the coefficients of system (33 - 36) are computed in [33]. The discussion of various solutions on the normal forms is nearly identical to the case (ii) above, except for the two additional first integrals, occurring as new constants in the discussion. The proofs for persistence are the same as before, due to a sufficiently large dimension of the space of symmetric points. So, here again, we have solutions homoclinic to exponentially small periodic waves. Let us notice that the above case was first treated in [114. The shape of the generalized solitary waves corresponding to these homoclinics is shown in figure 17. See 91 for a numerical computation of such waves.

Remark: If we add surface and/or interfacial tension, we have additional parameters. Bernoulli constants may appear as in system (4), and the sum of the number of these Bernoulli constants plus the dimension of the kernel of the linearized operator, is equal to the number of layers.

This allows more complicated bifurcations, with more eigenvalues appearing near the imaginary axis (in addition to the multiple 0 eigenvalue, or (and) to the corresponding freedom on Bernoulli constants). For example, in cases when a pair of double eigenvalues appears with a pair of simple eigenvalues on the imaginary axis, we cannot avoid, here again, exponentially small periodic oscillations at infinity for a class of generic situations (see 84). Moreover, in cases analogous to case (iii) $\left(00(i q)^{2}\right.$ resonance), for $q_{2}<0$, we have a large family of solutions homoclinic to each member of the family of reversible equilibria. In such a case there are solitary waves with exponentially damped oscillations at infinity, tending to constant flows, with the freedom on the velocity in each layer.

\section{Case of infinite depth}

The aim of this section is to present typical results for two-dimensional travelling waves in fluid layers when one layer (the bottom one) is infinitely deep. We consider in more detail the systems $(1,9),\left(1,7 \pi_{\infty}\right)$. We observed in $\S 3$ that the spectrum of the linear operator $L_{\mu}$ contains the full real line, and that, on it, the only possible eigenvalue is 0 . There are other eigenvalues $i k$ in the complex 
plane, solutions of the dispersion relation $\Delta[\mu, \operatorname{sgn}(\operatorname{Re} k) k]=0$.

In the case of system (19.9), we note an interesting situation where we have a pair of double eigenvalues $i k= \pm i / 2$ on the imaginary axis for $\mu_{1}=1 / 4$ [see (15)]. This is again a 1:1 resonance, but the rest of the spectrum is the full real line, which indeed crosses the imaginary axis, so we cannot use a center manifold reduction into an ODE (no gap between the imaginary axis and the rest of the spectrum). Notice that the point 0 in the spectrum is "resonant" with the purely imaginary eigenvalues, which may lead to problems even for the existence of periodic solutions.

In the case of system (1., $\mathbb{1}_{\infty}$ ), we note that 0 is always an eigenvalue with eigenvector $\xi_{0}^{\prime}$ [see (16) and $\$ 4.3$ ], and we have a pair of simple eigenvalues $i k= \pm i \lambda$ on the imaginary axis, and for $(1-\rho) \lambda \leq 1$ there are no other eigenvalues on the imaginary axis. For $(1-\rho) \lambda>1$ we have another pair $\pm i k_{1}$ of simple eigenvalues appearing on the imaginary axis, emerging from the continuous spectrum, and this new pair does not meet the other pair. For this system, interesting cases are the strong resonances when $k_{1} / \lambda=1 / 2$ or $1 / 3$, and a particularly interesting one is when $(1-\rho) \lambda$ is near 1 , when one pair of eigenvalues disappears (is melting) in the continuous spectrum.

\subsection{Periodic waves}

For periodic solutions, we follow the Lyapunov-Schmidt method, except that the presence of 0 in the spectrum gives some trouble (resonant terms). It appears that we can formulate all these problems, in such a way that there is no such resonant term. As a result, there are as many periodic solutions (with period near $\left.2 \pi / k_{0}\right)$ as in the truncated normal form (see [58/).

In this section, we consider the case of system (1. , $_{\infty}$ ) when there is a pair of simple imaginary eigenvalues $\pm i k_{0}$ of the operator $L_{\mu}$, such that other pairs $\pm i k_{1}$ which might be on this axis satisfy $k_{1} \neq n k_{0}$ for $n=1,2, \cdots$. This condition is satisfied in our case for $k_{0}=\lambda$, and is in general (for other similar problems) satisfied because there is only a finite number of eigenvalues on the imaginary axis, whose positions depend on the parameter set $\mu$. The method developed below assumes that the basic pair $\pm i k_{0}$ is not close to 0 , because this would imply that other pairs of eigenvalues would be close to some multiples of this pair.

It results that in general the only point in the spectrum of $L_{\mu}$ in resonance with our pair is 0 . The "exotic" character of this point of the spectrum leads to a specific difficulty we are dealing with below, by adapting the classical Lyapunov-Schmidt method for periodic orbits.

Let $s=\left(k_{0}+\gamma\right) x$, where $\gamma$ is close to 0 , and $k_{0}+\gamma$ is the wave number of the periodic solution we are looking for. We denote by $H_{\sharp}^{p}(E)$ the space of $(2 \pi$-periodic) functions of $s$, such that their derivatives up to order $p$ are in $L^{2}(\mathbb{R} / 2 \pi \mathbb{Z})$, taking values in the Banach space $E$. For such a space we use the norm defined by

$$
\|u\|_{H_{\sharp}^{p}}^{2}=\sum_{n \in \mathbb{Z}}\left(1+n^{2 p}\right)\left\|u_{n}\right\|_{E}^{2}
$$


which gives a Banach space structure. Let us define, in the space $\mathbb{H}_{\sharp}=H_{\sharp}^{1}(\mathbb{H})$, the linear operator $T_{\mu}=k_{0} \frac{d}{d s}-L_{\mu}$, with domain $\mathbb{D}_{\sharp}=H_{\sharp}^{2}(\mathbb{H}) \cap H_{\sharp}^{1}(\mathbb{D})$. The basic tool is the inversion of

$$
T_{\mu} U=V
$$

where $V$ is given in $\mathbb{H}_{\sharp}$, and where we look for $U \in \mathbb{D}_{\sharp}$. Now expanding in Fourier series $V$ and $U$ we have for $n \neq 0,1,-1$

$$
U_{n}=\left(i n \lambda \mathbb{I}-L_{\mu}\right)^{-1} V_{n},
$$

which, with the resolvent estimate (17), insures that if we define

$$
U^{\prime}=\sum_{n \in \mathbb{Z} \backslash\{0,1,-1\}} U_{n} e^{n i s} \in \mathbb{D}_{\sharp} \text {, then }\left\|U^{\prime}\right\|_{\mathbb{D}_{\sharp}} \leq C_{1}\|V\|_{\mathbb{H}_{\sharp}} \text { holds. }
$$

It then remains to study the equations $\left(i n k_{0} \mathbb{I}-L_{\mu}\right) U_{n}=V_{n}$ for $n=0,1,-1$. For $n=0$, the compatibility condition on $V_{0}$ and the additional condition for being in the range of $L_{\mu}$ are automatically satisfied by the $0^{\text {th }}$ Fourier component of the nonlinear term, for reversible solutions (in particular the nonlinear term has 0 components on $-\infty<y<0$ ). Then the 0 th Fourier component $U_{0}$ of the solution $U$ in (37) is uniquely determined in $\mathbb{D}$, up to an arbitrary multiple of the eigenvector $\xi_{0}^{\prime}$. For $n=1,-1$ this is a classical Fredholm alternative (one compatibility condition for $V_{1}$ and for $\left.V_{-1}\right)$.

Let us now consider the system (1, [1, $)_{\infty}$ ) rewitten as follows

$$
T_{\mu} U=G(\mu, \gamma, U)
$$

where we look for solutions in $\mathbb{D}_{\sharp}$, and

$$
G(\mu, \gamma, U)=-\gamma \frac{d U}{d s}+F(\mu, U)-L_{\mu} U .
$$

We observe that $G(\cdot, \cdot, \cdot)$ is analytic: $\mathbb{R}^{2} \times \mathbb{D}_{\sharp} \rightarrow \mathbb{H}_{\sharp}$ in the neighborhood of 0 , and is such that

$$
G(\mu, \gamma, 0)=0, \quad D_{(\gamma, U)} G(\mu, 0,0)=0 .
$$

Now, let us define the symmetry $\widehat{S}$ in $\mathcal{L}\left(\mathbb{H}_{\sharp}\right) \cap \mathcal{L}\left(\mathbb{D}_{\sharp}\right)$ by

$$
(\widehat{S} U)(s)=S U(-s) .
$$

It is then easy to check that $\widehat{S} T_{\mu}=-T_{\mu} \widehat{S}, \widehat{S} G(\mu, \gamma, U)=-G(\mu, \gamma, \widehat{S} U)$ holds.

For solving (39.40) we use a classical Lyapunov-Schmidt method. We are then able to prove the following (see proofs in [58]), where we denote by $\zeta_{\mu}$ the eigenvector of $L_{\mu}$ belonging to $i k_{0}$ :

Theorem 9 For $\left(u_{0},|A|\right)$ lying in a neighborhood of 0 , there is a family of periodic solutions of (1. T $_{\infty}$ ), bifurcating from 0 , which possess the following 
converging power series in $u_{0}, A, \bar{A}$ :

$$
\begin{aligned}
U(s) & =u_{0} \xi_{0}^{\prime}+A e^{i s} \zeta_{\mu}+\bar{A} e^{-i s} \bar{\zeta}_{\mu}+\sum_{n+p+q \geq 2} u_{0}^{n} A^{p} \bar{A}^{q} e^{i(p-q) s} U_{n p q} \in \mathbb{D}_{\sharp} \\
\gamma & =\sum_{n+r \geq 1} \gamma_{n r} u_{0}^{n}|A|^{2 r} \in \mathbb{R}, \\
s & =\left[k_{0}+\gamma\right] x .
\end{aligned}
$$

These solutions are reversible for $A$ real, and we have $S U_{n p q}=U_{n q p}=\bar{U}_{n p q}$. Moreover, we can find a unique analytic function $u_{0}=h\left(\mu,|A|^{2}\right)=O\left(|A|^{2}\right)$ such that at the interface, the averages of the velocities in the two fluids are equal (we can as well fix a Bernoulli first integral).

For other similar problems, the proofs are analogous, the number of arbitrary constants $u_{0}$ depending on the dimension of the kernel of $L_{\mu}$.

For cases of strong resonances, the additional difficulty is the same as in finite dimensional reversible systems. See for instance [71] for the study of periodic solutions for 1:2 resonance, and [58 for the 1:1 resonance. Roughly speaking, there are as many periodic solutions as would be given by the analysis of the corresponding generic reversible bifurcation in finite dimension.

A new difficulty occurs when one considers solutions with very large periods, for example in the case when the basic frequency is given by a pair of eigenvalues of $L_{\mu}$ close to 0 . The difficulty is that 0 also belongs to the spectrum (since it is on the real line), and that any other pair of imaginary eigenvalues is also quasi-resonant with the basic pair. This problem needs further investigations.

\subsection{Normal forms in infinite dimensions}

Since we cannot reduce our infinite depth problems to finite dimensional ODE's, we still would like to believe that eigenvalues near the imaginary axis are ruling the bounded solutions. This is a motivation for developing a theory of normal forms in separating the finite dimensional critical space from the rest (the "hyperbolic" part of the spectrum, including 0). This leads to "partial normal forms", where there are coupling terms, especially "bad" in the infinite dimensional part of the system (see [35, [65]). Indeed, there are some additional difficulties: i) when 0 is an eigenvalue embedded in the essential spectrum, we need the explicit form of the resolvent operator near the real axis, to extract the corresponding eigenmode from the rest of space, by a suitable projection (see [58, 66]), ii) in space $\mathbb{H}$ the linear operator does not have an "easy" (even formal) adjoint. This adjoint and some of its eigenvectors are usually necessary for expressing projections on the critical finite dimensional space. In our problems, we use again the explicit form of the resolvent operator near the (double) eigenvalues, to make the projections explicit (see [72]). Let us give below some details on these infinite dimensional normal forms.

Consider our system under the form

$$
\frac{d U}{d x}=F(\mu, U),
$$


where $F(\mu, 0)=0$ and $L_{\mu} U$ denotes its linear part. Assume that for $\mu=0$, one has a spectral decomposition of the form $\mathbb{H}=E_{0} \oplus E_{h}$ where $E_{0}$ is finite dimensional, and is spanned by all eigenvectors and generalized eigenvectors belonging to eigenvalues of $L_{0}$ lying on the imaginary axis. Then $E_{0} \subset \mathbb{D}$. The space $E_{h}$ is a complementary subspace invariant under $L_{0}$ which, in this subspace, has no eigenvalue on the imaginary axis, and is such that its spectrum contains the full real line (with no eigenvalue in 0 , and close to 0 ), and is bounded by a double sector in $\mathbb{C}$ centered on the real axis. In all the waterwave problems, with an infinitely deep bottom layer, we also have the estimate (17) for the resolvent of $L_{0}$ on the imaginary axis, far from 0 (this indeed implies the sectorial bound of the spectrum of $L_{\mu}$ for $\mu$ near 0 ). The above spectral decomposition of $\mathbb{H}$ implies that the 0 eigenvalue which occurs for instance in problem $\left(1,77_{\infty}\right)$ is extracted from the continuous spectrum, by using a suitable projection for being incorporated into $E_{0}$ (this is done explicitly in [58] and [66]). The result (see [65], 35]) is that one can find a polynomial change of variables in $\mathbb{H}$ such that

$$
U=V+W+\Phi(\mu, V, W), \quad V \in E_{0}, W \in E_{h},
$$

where $\Phi(\mu, \cdot, \cdot)$ is a polynomial with values in $\mathbb{D}$, of degree 1 in $W$ and degree $m$ in $V$, with regular coefficients in $\mu$, such that (41) reads

$$
\begin{aligned}
& \frac{d V}{d x}=L^{0} V+N(\mu, V)+R^{0}(\mu, V, W) \text { in } E_{0}, \\
& \frac{d W}{d x}=L^{h} W+R^{h}(\mu, V, W), \text { in } \mathbb{H},
\end{aligned}
$$

where $L^{0}=\left.L_{0}\right|_{E_{0}}, L^{h}=\left.L_{0}\right|_{E_{h}}$, and $N(\mu, V)$ corresponds to the usual finite dimensional polynomial (degree $m$ ) normal form, i.e. satisfies the following additional symmetry (see 62)

$$
e^{L^{0 *} x} N(\mu, V)=N\left(\mu, e^{L^{0 *} x} V\right), \quad \forall x \in \mathbb{R}, \forall V \in E_{0} .
$$

Now the remaining coupling terms satisfy the following estimates

$$
\begin{gathered}
\left\|R^{0}(\mu, V, W)\right\|_{E_{0}}=O\left(\|V\|^{m+1}+\|V\|\|W\|_{\mathbb{D}}+\|W\|_{\mathbb{D}}^{2}\right), \\
\left\|R^{h}(\mu, V, W)\right\|_{\mathbb{H}}=O\left[\left(\|V\|+\|W\|_{\mathbb{D}}\right)^{2}+\left|\mu\||| W\|_{\mathbb{D}}\right]\right.
\end{gathered}
$$

which means in particular that we have no linear term in $W$ in (42), and no linear term in $V$ in (43). Moreover, we observe that in general, there are quadratic terms in $V$ in (43), which determine the size of $W$ in this method. These quadratic terms are due to the resonance of the pure imaginary eigenvalues of $L_{0}$ with the point 0 in the continuous spectrum. Notice now that we simplify the system $(42,43)$ in using Bernoulli first integrals which allow to eliminate the coordinates belonging to the 0 eigenvalue. Indeed, this elimination is in general not singular with respect to $\mu$, however it becomes singular in the case of problem (1, $\left.7_{\infty}\right)$ when $(1-\rho) \lambda$ is close to 1 . Section $\S 5.4$ dealing with a 
new reversible bifurcation describes below what happens in such a case. It then appears that the above belief that eigenvalues on the imaginary axis govern bounded solutions, becomes wrong at least in this case. In fact our system (42,43) still contains all small bounded solutions of (41), but a scaling based on solutions corresponding to a truncated normal form in $V$ implies a "slaving" for $W$ which may not be satisfied for other types of solutions, such as the ones which occur in $\$ 5.4$.

\subsection{Results for the $(i q)^{2}$ resonance with continuous spec- trum}

With the method we use now, we need to give a priori the type of solution we are looking for. This is a major difference with the case where a (center manifold) reduction to an ODE is possible.

For periodic waves, with periods close to the basic one, one may use a method similar to the one given for the non resonant case, as it is done in [58]. We may also use the formulation (42,43), which is more transparent for such solutions, easily found on the reduced normal form in the variable $V$. The result is roughly that there are as many periodic solutions as in the finite depth case.

For solutions homoclinic to 0 (solitary waves), we first invert the infinite dimensional part (43) in $W$, using Fourier transform. Indeed, the linearized Fourier transform uses the resolvent operator of $L_{h}$. The fact that the resolvent operator is not analytic near 0 (0 is not a pole, since we eliminated it, but there is still a jump of the resolvent in crossing the real axis [65]) leads to the fact that this "hyperbolic part" of the solution cannot decay exponentially, but instead decays polynomially at infinity. The finite dimensional part (42) where $W$ is replaced by its expression in terms of $V$, is an integro-differential equation, because of the non local term coming from $W$. The principal part of this equation comes from the usual normal form. In the case of 1:1 resonance (for instance as in problem (1.9) for $\mu_{1}=1 / 4$, with double eigenvalues $i k=$ $\pm i / 2$ ), the normal form is given by (28) and in case of problem (11.9), $q_{2}<0$ holds (see 65). In other problems, with several layers, the 1:1 resonance may occur [except in problem $\left(1,7_{\infty}\right)$ ] with $q_{2}>0$ or $<0$ or even cancels (see [35]), leading to solutions similar to the ones given by the finite depth case, except that the convergence at infinity towards 0 or to a periodic wave is no longer exponential. For problem (1,9), and $\mu_{1} \gtrsim 1 / 4$, a fixed point argument in a space of polynomially decaying functions leads to the existence of two reversible homoclinics like in the finite dimensional case, except for the decay rate which is indeed proved to be in $1 / x^{2}$ in [11]. See also [1] where this decay is checked numerically.

The principal part of the solution at finite distance still comes from the finite dimensional truncated normal form, but it decays faster at infinity than the other part of the solution, which makes this tail part predominant at infinity. This is the main difference with the finite depth case, where the principal part coming from the normal form is valid for all values of $x$ [see 65] for the proofs related with problem (1, 9)]. 


\subsection{A new reversible bifurcation: pair of eigenvalues div- ing in the essential spectrum through the origin}

It appears that the technique described above may miss important solutions. This occurs precisely for the problem (1, $\left.\theta_{\infty}\right)$ when the parameter $\varepsilon=1-(1-\rho) \lambda$ is close to 0 . In such a case the singularity of the resolvent operator $\left(i q \mathbb{I}-L_{\mu}\right)^{-1}$ is a little worse when $\varepsilon \rightarrow 0$. Indeed the projection operator on the eigenvector belonging to the 0 eigenvalue becomes singular, having $\varepsilon$ in its denominator! One may then suspect that this changes relative orders of magnitudes for various components of the variable $U$. The dispersion relation (16) in this case shows a first factor giving two isolated eigenvalues $i k= \pm i \lambda$, and for $k$ real near 0 , a second factor such that $|k|\left[\varepsilon+\rho|k|+O\left(|k|^{3}\right)\right]$ giving the 0 eigenvalue, and a pair of imaginary eigenvalues near 0 only for $\varepsilon<0$. For $\varepsilon>0$, the factor of $|k|$ corresponds to the linear part of the dispersion relation for the BenjaminOno model (see 16], 31, [95]). Indeed, a suitable scaling here allows to find an asymptotic expansion in powers of $\varepsilon$ of a formal solitary wave, with a decay in $1 / x^{2}$ at infinity [66], with the same principal part as the solitary wave solution of the Benjamin-Ono equation. In addition, as shown above, we also obtain a two parameter family of bifurcating periodic waves (their amplitude is one of the parameters) with periods close to $2 \pi / \lambda$. We can use the form of the family of periodic solutions (given in theorem 1) to construct $\Phi$ in the change of variables leading to the normal form (42,43). It then appears that the manifold $W=0$ contains all the family of periodic solutions, and that the $W$ part of the system possesses an approximate homoclinic to 0, close to the Benjamin-Ono solitary wave. Finally, the formal solitary wave is not a solution of $\left.(1,7]_{\infty}\right)$, because of the additional frequency $\lambda$. It is shown in 66 , that there are two reversible solutions homoclinic to each of the above periodic wave, provided that their amplitude is not too small (exponentially small in $\varepsilon$ as it is proved in 85]). The principal part at finite distance of all these "generalized solitary waves" is given by the approximate homoclinic described above (see figure 17). One difficulty here is that it is not possible to "morally" reduce the problem to a finite dimensional one, as in 82], [34, 109, 113, because of the essential spectrum filling the real axis (especially near 0). Another difficulty, not appearing in previous work, is that the decay at infinity, towards the periodic solution (with a shift opposite at both infinities), is only polynomial, instead of exponential. This implies the use of refinement techniques for being able to use a fixed point technique in a good function space. See [96] for a numerical computation of periodic as well as generalized solitary waves.

Notice that if we consider the problem of two superposed layers, the bottom one being, as here, infinitely deep, but the top layer being bounded by a rigid horizontal wall, we may formulate the problem as a dynamical system, in a simpler way than here, and the spectrum of the linearized operator is simplified, in the sense that we do not have the pair of eigenvalues $\pm i \lambda$ on the imaginary axis. This avoids the problems above provoked by the additional frequency, and one finds a solution homoclinic to 0, close to the Benjamin-Ono solitary wave (see [3],112] for proofs, not relying on a dynamical system formulation). 


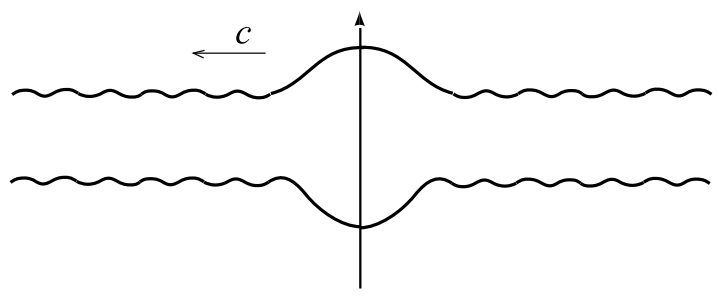

Figure 17: Shape of the generalized solitary waves for a two-layer system.

\section{Stratified fluids}

A large class of 2D travelling waves in perfect fluids appears as internal waves in a stratified medium. A simplified model of a stratified layer consists in considering two (or more) superposed immiscible fluids of different densities. The interface between two fluids is then an unknown and an analysis in the form of a reversible dynamical system may be performed as we indicated in section 2.3 , where we replace the upper free surface by a rigid boundary (same boundary condition as at the bottom, see for instance Amick and Turner [9], 10], and see Mielke [93] for a formulation as a dynamical system). In fact, a more realistic version of the problem is when the density $\rho$ increases continuously from top to bottom, as a known function of the stream function $\Psi(d \rho / d t=0$ and incompressibility i.e. $\operatorname{div} U=0$ ). Basic papers in this respect are [2], [118], [17]. There are various ways of formulating this problem as a dynamical system. For instance, a popular one consists in considering the equation satisfied by $\Psi$, named the Dubreil-Jacotin-Long equation (DJL) 38

$$
\begin{aligned}
\Delta \Psi+\left(\lambda z+\frac{|\nabla \Psi|^{2}}{2}\right) \frac{\rho^{\prime}(\Psi)}{\rho(\Psi)} & =\left(\lambda \Psi+\frac{1}{2}\right) \frac{\rho^{\prime}(\Psi)}{\rho(\Psi)}, \\
\left.\Psi\right|_{z=0,1} & =0
\end{aligned}
$$

in the strip $\{0<z<1\}$, which may be written easily in a dynamical system form, and in which a bifurcation similar to case (i) of section 4.2.1 occurs (see [75]), and a bifurcation to fronts (see [70]) connecting two uniform states, resulting from the cancellation of the quadratic coefficient $a$ in (24). There are results on periodic internal waves and large amplitude solutions in 78 , and a study of 3D internal flows, with a derivation of the K-P model (see 18), and a dimension breaking bifurcation in [79, in the spirit mentioned in section 7.4 below. An interesting problem arises as one tends towards the limiting case when the density stratification becomes discontinuous, i.e. when $\rho$ has a large gradient in a small region near a certain height $h$, and tends to a piecewise constant $\rho_{0}$, having a discontinuity in $h$. We see on the DJL equation that the factor $\rho^{\prime} / \rho$ becomes singular, and the limit is to be understood in the distribution sense. A unified formulation, including the discontinuous case (a free interface separates two fluids with different constant densities) as well as the continuous stratifica- 
tion case, is proposed by G.James [69]. He studies the limit $\rho(\Psi) \rightarrow \rho_{0}$, where $\rho_{0}(z)=1$ for $z \in(0, h)$, and $\rho_{0}(z)=q<1$ for $z \in(h, 1)$, and $\rho(\Psi)$ is a decreasing function such that $\rho(0)=1, \rho(1)=q$, with $\left|\rho^{\prime}(\psi)\right|$ becoming very large in $(h-\varepsilon, h+\varepsilon)$. His formulation uses the coordinates $(x, \psi)$ and the unknown function $Y=Z(x, \psi)-\psi$, where by definition $\Psi[x, Z(x, \psi)] \equiv \psi$. The system becomes

$$
\begin{aligned}
& \frac{\partial Y}{\partial x}=\frac{U}{\rho}\left(1+\frac{\partial Y}{\partial \psi}\right), \\
& \frac{\partial U}{\partial x}=\lambda \rho^{\prime} Y+\frac{1}{2} \frac{\partial}{\partial \psi}\left\{\rho\left[\left(1+\frac{\partial Y}{\partial \psi}\right)^{-2}-1\right]+\frac{U^{2}}{\rho}\right\},
\end{aligned}
$$

with boundary conditions $\left.Y\right|_{\psi=0,1}=0$. In (44), $U=\rho v$ ( $v$ is the vertical component of the velocity), and $\frac{\partial Y}{\partial x}=v / u$ is the slope of a streamline. This system is reversible under the symmetry $(Y, U) \mapsto(Y,-U)$. In the case $\rho=\rho_{0}$, we have $\rho_{0}^{\prime}=-(1-q) \delta_{h}$ where $\delta_{h}$ is the Dirac distribution in $\psi=h$. Here the basic spaces are

$$
\begin{aligned}
& \mathbb{H}=W_{h}^{1,1} \times L_{h}^{1}, \\
& \mathbb{D}=\left\{(Y, U) \in W_{h}^{2,1} \times W_{h}^{1,1} ; Y(0)=Y(1)=0\right\},
\end{aligned}
$$

where

$$
\begin{aligned}
L_{h}^{1} & =L^{1}(0,1) \oplus \mathbb{C} \delta_{h}, \\
W_{h}^{n, 1} & =\left\{u \in W^{n-1,1}(0,1) ;\left.u\right|_{(0, h)} \in W^{n, 1}(0, h),\left.\quad u\right|_{(h, 1)} \in W^{n, 1}(h, 1)\right\},
\end{aligned}
$$

and where we notice that $\mathbb{D}$ is not dense in $\mathbb{H}$. James shows that a solution in $C^{0}(\mathbb{R}, \mathbb{D}) \cap C^{1}(\mathbb{R}, \mathbb{H})$ of this system leads to a classical solution in the continuous stratification case, and leads to the standard discontinuous case (no interfacial tension) in the $\rho_{0}$ case. This formulation is weak in the sense that the dependency in $\rho$ of operators is weakly continuous, despite the fact that $\int_{0}^{1}\left|\rho^{\prime}(\psi)\right| d \psi=1-q$ is a finite constant. The author is able to prove an estimate like (17) on the resolvent of the linearized operator, uniformly in $\rho$. This allows the existence of a family of center manifolds in a ball of $\mathbb{D}$ centered at 0 , this ball being independent of $\rho$ close to $\rho_{0}$. Here the result of Kirrmann for finding center manifolds is needed [76], because one works in Banach spaces. The dependency in $\rho$ on these center manifolds is only continuous in $\mathbb{H}$ (not in $\mathbb{D})$. However, if one considers a typical bifurcation problem like the one studied in case (i) of section 4.2.1, one obtains a two-dimensional amplitude equation, whose coefficients are functions of the stratification $\rho$. James shows that the vector field as well as these coefficients, at any order, are continuous functions of $\rho$, as $\rho \rightarrow \rho_{0}$ (topology of $L^{2}$ ). These technical results are proved in 69.

\section{Three-dimensional travelling water waves}

We first consider 3D water waves that bifurcate from the state of rest, and for the sake of brevity, we only expose here in detail the case of waves which are 
periodic in the direction $y$ orthogonal to the direction $x$ of propagation, which is the reversible version of the paper of Groves \& Mielke 47] based on a hamiltonian formulation (see also Bridges [19]). In the cases where the waves are periodic along the direction of propagation ([45], [53]), the technique is similar. We assume that surface tension is present, which seems to be fundamental in the present formulation for allowing a finite dimensional reduction process. We thank Mariana Hărăgus for giving us the 3D formulation indicated below. Most papers which present a dynamical system approach use a hamiltonian formulation of the problem ( 45, 446, 447]). Notice that no rigorous mathematical results exist so far on the Euler equations in the case of travelling water waves which are localized in all horizontal directions. Notice also that for the study of travelling waves which are periodic in both horizontal directions, there is no need for a dynamical system formulation (see 100, 29]). In section 7.4 we give some results on the "dimension breaking" bifurcations, which consist in the bifurcation of $3 \mathrm{D}$ waves from $2 \mathrm{D}$ waves of finite amplitude.

\subsection{Formulation as a dynamical system}

One of the difficulties is that, even for one-layer systems (one rigid bottom, one free surface), there are no simple variables providing a flattening of the free surface, as it is the case for the $2 \mathrm{D}$ case. Formulating the problem in the moving frame, with velocity $-c$ along the $x$ direction, we are interested in steady solutions close to the uniform flow of speed $c$ ( 1 in dimensionless variables) in the $x$ direction. Let us use the velocity potential $x+\phi$, where $\phi$ is the perturbation potential. We define as before for system (4), the dimensionless parameters $\lambda$ and $b$. We denote by $z$ the vertical direction. Then the rigid bottom is located at $z=0$, while we denote by $z=1+\eta(x, y)$ the equation of the free surface (the depth $h$ is the length scale), where $\eta$ is close to 0 . Then the problem satisfies the following system

$$
\begin{gathered}
0=\phi_{x x}+\phi_{y y}+\phi_{z z}, \quad 0<z<1+\eta(x, y) \\
0=\phi_{z} \text { on } z=0, \\
0=\eta_{x}+\eta_{x} \phi_{x}+\eta_{y} \phi_{y}-\phi_{z}, \text { on } z=1+\eta(x, y) \\
C=\phi_{x}+\frac{1}{2}\left(\phi_{x}^{2}+\phi_{y}^{2}+\phi_{z}^{2}\right)+\lambda \eta+ \\
-b\left[\frac{\eta_{x}}{\left(1+\eta_{x}^{2}+\eta_{y}^{2}\right)^{1 / 2}}\right]_{x}-b\left[\frac{\eta_{y}}{\left(1+\eta_{x}^{2}+\eta_{y}^{2}\right)^{1 / 2}}\right]_{y}, \quad \text { on } z=1+\eta(x, y),
\end{gathered}
$$

where $C$ is the Bernoulli constant on the free surface, and we notice that the factor of $-b$ is twice the mean curvature of the free surface. Let

$$
u=\phi_{x}, \quad \xi=\frac{\eta_{x}}{\left(1+\eta_{x}^{2}+\eta_{y}^{2}\right)^{1 / 2}} .
$$

Hence our system may be rewritten as follows (where $\Delta$ denotes the Laplace operator in the $(y, z)$ plane)

$$
\left.\begin{array}{l}
\phi_{x}=u \\
u_{x}=-\Delta \phi,
\end{array}\right\} \text { for } 0<z<1+\eta(x, y)
$$




$$
\begin{gathered}
\eta_{x}=\xi\left(\frac{1+\eta_{y}^{2}}{1-\xi^{2}}\right)^{1 / 2}, \\
\xi_{x}=\frac{1}{b} u+\frac{\lambda}{b} \eta+\frac{1}{2 b}\left(u^{2}+\phi_{y}^{2}+\phi_{z}^{2}\right)-\left[\eta_{y}\left(\frac{1-\xi^{2}}{1+\eta_{y}^{2}}\right)^{1 / 2}\right]_{y}+c_{1}, \text { on } z=1+\eta(x, y)
\end{gathered}
$$

where $c_{1}=-C / b$, and with the boundary conditions

$$
\begin{aligned}
\phi_{z} & =0 \text { on } z=0 \\
\phi_{z} & =\xi(1+u)\left(\frac{1+\eta_{y}^{2}}{1-\xi^{2}}\right)^{1 / 2}+\eta_{y} \phi_{y} \text { on } z=1+\eta(x, y) .
\end{aligned}
$$

We now flatten the upper boundary in making the change of coordinates

$$
z^{\prime}=\frac{z}{1+\eta(x, y)} \text {. }
$$

It follows that $z^{\prime} \in(0,1)$. In the new coordinate system $\left(x, y, z^{\prime}\right)$, we find after dropping the primes

$$
\begin{gathered}
\phi_{x}=u+\frac{z \phi_{z} \xi}{1+\eta}\left(\frac{1+\eta_{y}^{2}}{1-\xi^{2}}\right)^{1 / 2} \\
u_{x}=-\left(\phi_{y}-\frac{z \eta_{y}}{1+\eta} \phi_{z}\right)_{y}+\frac{z \eta_{y}}{1+\eta}\left(\phi_{y}-\frac{z \eta_{y}}{1+\eta} \phi_{z}\right)_{z}-\frac{\phi_{z z}}{(1+\eta)^{2}}+\frac{z u_{z} \xi}{1+\eta}\left(\frac{1+\eta_{y}^{2}}{1-\xi^{2}}\right)^{1 / 2}, \\
\eta_{x}=\xi\left(\frac{1+\eta_{y}^{2}}{1-\xi^{2}}\right)^{1 / 2}, \\
\xi_{x}=\frac{1}{b}\left(\left.u\right|_{z=1}+\lambda \eta\right)+\frac{1}{2 b}\left[u^{2}+\left(\phi_{y}-\frac{z \eta_{y}}{1+\eta} \phi_{z}\right)^{2}+\frac{\phi_{z}^{2}}{(1+\eta)^{2}}\right]_{z=1}^{+} \\
-\left[\eta_{y}\left(\frac{1-\xi^{2}}{1+\eta_{y}^{2}}\right)^{1 / 2}\right]_{y}+c_{1}
\end{gathered}
$$

with boundary conditions

$$
\begin{aligned}
\left.\phi_{z}\right|_{z=0} & =0 \\
\left.\phi_{z}\right|_{z=1} & =\left(\frac{1+\eta}{1+\eta_{y}^{2}}\right)\left[\xi\left(1+\left.u\right|_{z=1}\right)\left(\frac{1+\eta_{y}^{2}}{1-\xi^{2}}\right)^{1 / 2}+\left.\eta_{y} \phi_{y}\right|_{z=1}\right] .
\end{aligned}
$$


We need to define suitable function spaces for the dynamical system formulation, and then we shall need to modify the boundary condition, to put it into a linear form. Define

$$
H_{\sharp, P}^{s}=\left\{f \in H_{l o c}^{s}(\mathbb{R}) ; f(y+P)=f(y), \text { for almost all } y \in \mathbb{R}\right\},
$$

$H_{\sharp}^{s}(\Sigma)=\left\{f \in H_{\text {loc }}^{s}[\mathbb{R} \times(0,1)] ; f(y+P, z)=f(y, z)\right.$, for almost all $\left.(y, z) \in \mathbb{R} \times(0,1)\right\}$,

where $H^{s}$ is the classical Sobolev space, and in which $P>0$ is the period of the waves in $y, \Sigma=(0, P) \times(0,1)$ and we assume $0<s<1 / 2$. Now set

$$
\mathbb{H}_{s}=H_{\sharp}^{s+1}(\Sigma) \times H_{\sharp}^{s}(\Sigma) \times H_{\sharp, P}^{s+1} \times H_{\sharp, P}^{s} .
$$

The system above is of the form

$$
\frac{d W}{d x}=\widetilde{F}(W)
$$

with

$$
W=(\phi, u, \eta, \xi),
$$

and $\widetilde{F}: \mathbb{H}_{s+1} \rightarrow \mathbb{H}_{s}$ is a smooth function with domain (codimension-two manifold in a neighborhood of 0 )

$$
\begin{aligned}
& D(\widetilde{F})=\left\{(\phi, u, \eta, \xi) \in \mathbb{H}_{s+1} ;|\xi|<1, \eta>-1,\left.\phi_{z}\right|_{z=0}=0\right. \\
& \left.\left.\quad \phi_{z}\right|_{z=1}=\left(\frac{1+\eta}{1+\eta_{y}^{2}}\right)\left[\xi\left(1+\left.u\right|_{z=1}\right)\left(\frac{1+\eta_{y}^{2}}{1-\xi^{2}}\right)^{1 / 2}+\left.\eta_{y} \phi_{y}\right|_{z=1}\right]\right\}
\end{aligned}
$$

Remark that $H_{\sharp, P}^{s}$ and $H_{\sharp}^{t}(\Sigma)$ are Banach algebras for $s>1 / 2, t>1$, and that $H_{\sharp, P}^{s+1} \cdot H_{\sharp, P}^{s} \subset H_{\sharp, P}^{s}$, and $H_{\sharp}^{s+1}(\Sigma) \cdot H_{\sharp}^{s}(\Sigma) \subset H_{\sharp}^{s}(\Sigma)$ if $s>0$.

One of the difficulties here is the incorporation of a nonlinear boundary condition in the function space! Let us now transform the boundary conditions into linear ones. To do this, we define in a neighborhood of 0 a smooth function $H: \mathbb{H}_{s+1} \rightarrow H_{\sharp}^{s+1}(\Sigma)$ by

$$
H(\phi, u, \eta, \xi)=z\left(\frac{1+\eta}{1+\eta_{y}^{2}}\right)\left[\xi(1+u)\left(\frac{1+\eta_{y}^{2}}{1-\xi^{2}}\right)^{1 / 2}+\eta_{y} \phi_{y}\right] .
$$

Then the boundary conditions become

$$
\phi_{z}=H(\phi, u, \eta, \xi) \text { on } z=0,1 .
$$

We make the change of variables near 0 , using $G: \mathbb{H}_{s+1} \rightarrow \mathbb{H}_{s+1}$ given by

$$
G(\phi, u, \eta, \xi)=\left(\phi-\varphi_{z}, u, \eta, \xi\right)
$$

where $\varphi \in H_{\sharp}^{s+3}(\Sigma)$ is the unique solution of the linear boundary value problem

$$
\begin{aligned}
\Delta \varphi & =H(\phi, u, \eta, \xi)-z \xi, \quad(y, z) \in \Sigma \\
\varphi & =0 \text { on } z=0,1 .
\end{aligned}
$$


We now define

$$
\psi=\phi-\varphi_{z} .
$$

Hence the boundary conditions become

$$
\psi_{z}=0 \text { on } z=0,1
$$

It can be shown that $G$ defines a change of variables near 0 , by checking that its (bounded) inverse at the origin is $[d G(0)]^{-1}=\mathbb{I}$. It can be shown (as in 47 ) that the operator $d G(U): \mathbb{H}_{s+1} \rightarrow \mathbb{H}_{s+1}$ extends to an isomorphism $\widetilde{d G}(U)$ : $\mathbb{H}_{s} \rightarrow \mathbb{H}_{s}$ for every $U$ near 0 in $\mathbb{H}_{s+1}$.

Now set $W=G^{-1}(U)$ in 45 , and find the new system for $U=G(W)$ which reads

$$
\frac{d U}{d x}=d G\left[G^{-1}(U)\right]\left\{\widetilde{F}\left[G^{-1}(U)\right]\right\},
$$

where $U=G(\phi, u, \eta, \xi)=(\psi, u, \eta, \xi)$ as defined above satisfies the boundary conditions $\psi_{z}=0$ on $z=0,1$. Finally, our system may be written as

$$
\frac{d U}{d x}=F(\mu, U)
$$

as before, where $\mu=\left(\lambda, b, c_{1}, P\right)$ and $F$ is smooth acting from $\mathbb{D}_{s}=\{(\psi, u, \eta, \xi) \in$ $\left.\mathbb{H}_{s+1} ;\left.\psi_{z}\right|_{z=0,1}=0\right\}$ into $\mathbb{H}_{s}$. Notice that $\mathbb{D}_{s}$ is dense in $\mathbb{H}_{s}$ since $s<1 / 2$ (this density is useful to avoid "technical complications" in the reduction procedure).

\subsection{Spectrum of the linearized operator}

Let us proceed as in 47. The linearized operator at the origin (solution for $\left.c_{1}=0\right)$ reads

$$
L_{\mu}=d \widetilde{F}(0) \text {, }
$$

and acts in $\mathbb{H}_{s}$ with domain $\mathbb{D}_{s}$. The linear operator $L_{\mu}$ is then defined by

$$
d \widetilde{F}(0)(\phi, u, \eta, \xi)=\left(u,-\Delta \phi, \xi, b^{-1}\left(\left.u\right|_{z=1}+\lambda \eta\right)-\eta_{y y}\right)
$$

with boundary conditions

$$
\left.\phi_{z}\right|_{z=0}=0,\left.\quad \phi_{z}\right|_{z=1}=\xi
$$

The eigenvalues $\sigma$ of $L_{\mu}$ satisfy the dispersion relation

$$
\left(\lambda-b \tau_{n}^{2}\right) \tau_{n} \sin \tau_{n}-\sigma^{2} \cos \tau_{n}=0,
$$

where

$$
\tau_{n}^{2}=\sigma^{2}-\frac{4 \pi^{2} n^{2}}{P^{2}}, \quad n \in \mathbb{N} .
$$

For $n=0$ and $\sigma=i k$ we naturally recover the $2 \mathrm{D}$ dispersion relation (12). We may observe that $\sigma=0$ is a double eigenvalue with the eigenmode $(1,0,0,0)$, and the generalized eigenmode $(0,1,-1 / \lambda, 0)$ due to the invariance of the system 


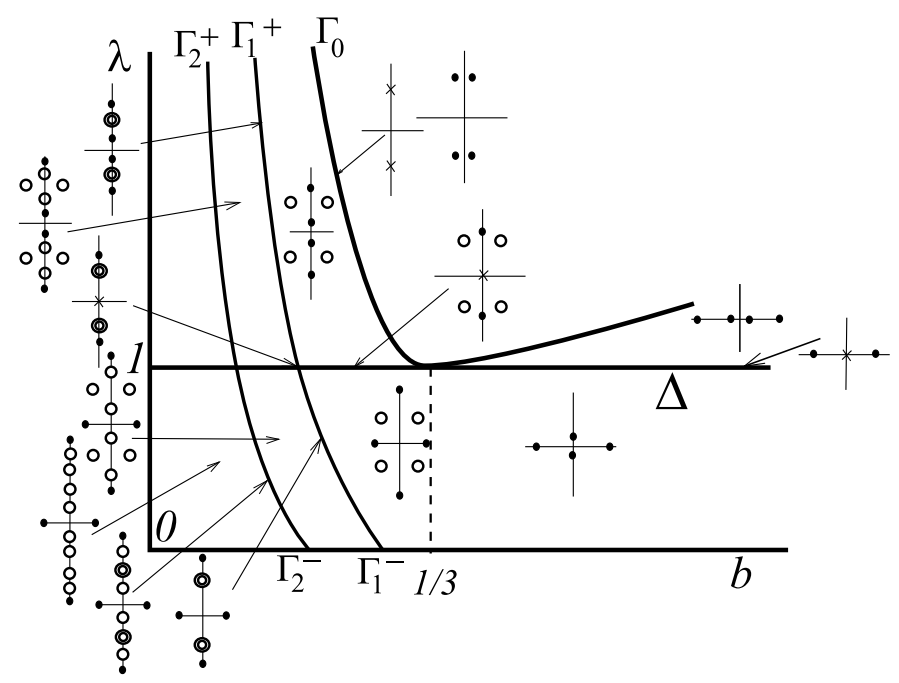

Figure 18: The dots and circles represent respectively 2D and 3D eigenvalues. Crosses are double (2D) eigenvalues, double circles are quadruple (3D) eigenvalues. Compare with figure 5 (2D case).

under the shift $\phi \rightarrow \phi+$ const and to the freedom on the Bernoulli constant, which leads to a two-parameter family of trivial solutions. The eigenmode may be eliminated by fixing an additional linear condition on $\phi$, and we may fix the Bernoulli constant for eliminating completely the eigenvalue 0. Moreover, the system has an $O(2)$ symmetry invariance, due to translational invariance in $y$, added to periodicity, and to the reflection symmetry $y \rightarrow-y$. It results that for $n \neq 0$, all eigenvalues are at least double (with a factor $e^{ \pm 2 i \pi n y / P}$ in the eigenmode). Moreover, as shown in [47], the spectrum of $L_{\mu}$ consists entirely of isolated eigenvalues of finite multiplicities (the above ones), and we also have the estimate (17) on the resolvent (the proof in $\mathbb{H}_{0}$ is simpler than in 47 ). The location of "critical" eigenvalues is summerized in figure 18 (see [50], 47]). A center manifold reduction is possible as indicated in section 3.3, using the method of [92] or [76]. The study of the solutions given by the normal form and of their persistence under remaining high order terms is made in 447. In addition to waves periodic in $x$ and $y$, waves periodic in $y$ and quasi-periodic in $x$, and generalized solitary waves, periodic and even in $y$, tending at infinity to periodic waves in the propagation direction $x$, can be found. We refer to this paper for getting the detailed form of these solutions, whose study is analogous to the two-dimensional case, except for the high dimension of the center manifold (restricted to even solutions in $y$ in 47) which leads to discussions on high dimensional reversible ODEs. A typical study of the normal form (in a tendimensional reduced space) without restrictions on the symmetry of solutions is made by Hărăguş \& Il'ichev in [50]. It corresponds to the bifurcation occuring 
near the curve $\Gamma_{1}^{-}$of figure 18 where a simple pair of eigenvalues and a pair of quadruple eigenvalues $(2 \times 2$ Jordan blocks $)$ sit on the imaginary axis.

\subsection{Three-dimensional travelling waves periodic in the di- rection of propagation}

For waves periodic in the direction of propagation $x$, with an arbitrary profile in $y$, the formulation may follow the same method as above. This was done by Groves in 445, who used a hamiltonian formulation. It can also be done in the framework of reversible vector fields like in [53. Here again we have an $O(2)$ symmetry, due to $x \rightarrow-x$ invariance of the problem, and 0 is still at least a double eigenvalue, for the same reasons as above. The picture for critical eigenvalues near the imaginary axis depends on the same set of parameters $\left(\lambda, b, P, c_{1}\right)$, but it looks quite different from the one given in figure 18, see [45] for details (case $c_{1}=0$ ). In 45] and [53, the authors study the first non trivial $3 \mathrm{D}$ bifurcation, where the reduced space is 6 -dimensional due to a pair of real double (3D) eigenvalues meeting at 0 (already double). The full normal form is studied in [53]. In [45] the author obtains large families of periodic and quasiperiodic solutions. Restricting the study to even solutions in $x$, 45. and 53. reduce the dimension to 2 . This leads to an analysis similar to the one made in section 4.1 (the shift invariance $\phi \rightarrow \phi+$ const may be eliminated easily), with a parity symmetry in addition which cancels quadratic terms in the normal form. They can then prove the existence of an homoclinic solution corresponding to a wave periodic in $x$, and localized (and even) in $y$. We refer to 45. and 53. for details of the proof. In addition, there is in [53] a discussion on the next bifurcation (eight dimensional reduction), which reduces, for even solutions, to the same case (ii) as in section 4.2.2.

\subsection{Dimension breaking bifurcation}

This topic first appeared in Hărăguş-Kirchgässner [51]. The idea is to look for bifurcations of 3D travelling waves from a non trivial $2 \mathrm{D}$ travelling wave. The difficulty is to determine the spectrum of the linearization about the $2 \mathrm{D}$ wave, since the linear operator has $x$ - dependent coefficients, so that the spectrum cannot be calculated explicitly - except in some very special cases involving model equations (see [32, 52, 54, 20]).

The only existing work at this time on the "real" water-wave problem, is by Groves, Hărăguş \& Sun 46]. The authors start with the $2 \mathrm{D}$ solitary waves occuring when $b>1 / 3$ (see section 4.2.1), and consider bifurcating waves, periodic in the transverse direction $y$. They study the linearized operator (this part is technical and more complicated than in model equations), and show the existence of a pair of pure imaginary eigenvalues close to 0,0 itself belonging to the essential spectrum. It is then possible to show the bifurcation of a family of solutions periodic in $y$, in a way analogous to the one mentioned in section 5.1. The family of solutions they obtain cannot be obtained via the analysis of 
section 7.2 , in starting directly from the 0 solution. We should notice that the above method does not give any fully localized solutions.

\section{Two-dimensional standing wave problem}

In contrast with the other sections, we consider in this section the standing wave problem, i.e. non travelling waves, which are supposed to be periodic in the horizontal direction and in time, with a vertical mirror symmetry. This old problem, which goes back to Stokes [108], has received considerable interest recently. The aim of this section is to indicate the most recent results. The notations here are selfconsistent.

Let us denote by $-\underline{h}<y<\eta(x, t)$ the region occupied by the liquid, where $\eta$ is the height of the free surface ( 0 when the fluid is at rest) and $\underline{h}$ the average depth; we assume that the flow is potential, and we denote the potential by $\phi(x, y, t)$. We look for time periodic (period $T$ ), and $x$-periodic flows (wave length $\lambda$ ). Choosing respectively $T / 2 \pi, \lambda / 2 \pi, \lambda / T, \lambda^{2} / 2 \pi T$ as scales of time, length, velocity and potential, we obtain the dimensionless system of equations (below $\underline{h}=h \lambda / 2 \pi$ )

$$
\begin{gathered}
\Delta \phi=0, \quad \text { in }-h<y<\eta(x, t) \\
\left.\begin{array}{r}
\frac{\partial \eta}{\partial t}+u \frac{\partial \eta}{\partial x}-v=0 \\
\frac{\partial \phi}{\partial t}+\frac{1}{2}\left(u^{2}+v^{2}\right)+\mu \eta=0
\end{array}\right\} \text { on } y=\eta(x, t), \\
\frac{\partial \phi}{\partial y}=0 \text { on } y=-h
\end{gathered}
$$

where the velocity components $(u, v)$ satisfy $u=\partial \phi / \partial x, v=\partial \phi / \partial y$, and where $\mu=g T^{2} / 2 \pi \lambda, g$ being the acceleration due to gravity. Restricting the study to solutions with $\eta$ even in $t$ and in $x$, the linearization of $(47,48,49$ ) near 0 (a flat free surface) gives solutions of the form

$$
\begin{aligned}
\eta(x, t) & =\cos q t \cos p x, \\
\phi(x, y, t) & =-\frac{q}{p \sinh (p h)} \sin q t \cos p x \cosh p(y+h),
\end{aligned}
$$

provided that the dispersion relation

$$
\mu p \tanh (p h)=q^{2}
$$

is satisfied. Assume that we choose $\mu_{0}$ and $h_{0}$ such that (50) has a unique positive solution $(p, q)=\left(p_{0}, q_{0}\right) \in \mathbb{N}^{2}$. Then, we observe that even though $\left|\mu p \tanh (p h)-q^{2}\right| \neq 0$ for $(p, q) \neq\left(p_{0}, q_{0}\right)$, this quantity may be arbitrarily small (leading to a small divisor problem, mentioned below). The structure of the nonlinear problem may be set into the form

$$
\mathcal{F}(X, \mu, h)=0
$$


where $X$ lies in a suitable function space $(2 \pi-$ periodic in $t$ and in $x)$, and where $\mathcal{F}$ is analytic in its arguments, taking values in another suitable function space. The first step is to transform the original problem into such a formulation (especially fixing the domain). This was done in 99] by using Lagrangian coordinates, while in the infinite depth case it is done via a conformal mapping in [104], [8]. The known solution $X=0$ corresponds to the fluid at rest, and we have a linearized operator

$$
L_{0}=D_{X} \mathcal{F}\left(0, \mu_{0}, h_{0}\right)
$$

having a one dimensional kernel. If the problem was an ordinary bifurcation problem, there would be a non trivial continuous branch $\{X(\varepsilon), \mu(\varepsilon), h(\varepsilon)\}$ of solutions of (51), where $\mu(0)=\mu_{0}, h(0)=h_{0}$. In fact, the pseudo-inverse $\widetilde{L}_{0}^{-1}$ of $L_{0}$ (inverse on its range) is not bounded, because of the small divisor problem. We then need to restrict the parameter set to values where suitable diophantine conditions are realized, for having a bound for $\widetilde{L}_{0}^{-1}$ (controling the loss of regularity). Moreover, since we need to use the Nash-Moser implicit function theorem, we also need to be able to invert (with a uniform bound for the inverse) the linearized operator at a non zero $X$, which is much more difficult, and in particular, again requires diophantine conditions to be realized. All this is done in the work of Plotnikov and Toland [99. Their result is valid for $(\mu, h)$ near $\left(\mu_{0}, h_{0}\right)$ satisfying conditions which are described below.

First, one defines for $\nu \in(1,2)$,

$$
\mathcal{N}(\nu)=\left\{\mu>0 ;\left.\exists c(\mu, \nu)\left|q^{2}-\mu\right| p\right|^{-1} \leq c(\mu, \nu)(1+|q|)^{\nu}, \forall(p, q) \in \mathbb{Z}^{2} \backslash\{0\}\right\}
$$

and one defines for any fixed $\mu$ the set

$$
\Sigma(\mu)=\left\{h>0 ; q^{2}-\mu p \tanh (p h)=0 \text { has at least one solution }(p, q) \in \mathbb{N}^{2}\right\} .
$$

Then it is shown that $\mathcal{N}(\nu)$ has full measure, and that for $\mu_{0} \in \mathcal{N}(\nu)$, the set $\Sigma\left(\mu_{0}\right)$ is countable with only one limit point, namely 0 . Now we define the subset $\mathcal{N}_{0}(\nu) \subset \mathcal{N}(\nu)$ such that the equation $q^{2}-\mu p \tanh (p h)=0$ has at most one positive integer solution for any $\mu_{0} \in \mathcal{N}_{0}(\nu)$ and any $h_{0}$. It is shown that the complement of $\mathcal{N}_{0}(\nu)$ in $(0, \infty)$ has zero measure. The result is the following (see 99 for a more precise statement):

Theorem 10 (Plotnikov-Toland) Choose $\nu \in(1,24 / 23), \mu_{0} \in \mathcal{N}_{0}(\nu), h_{0} \in$ $\Sigma\left(\mu_{0}\right)$, and denote by $(p, q)$ the unique solution of $q^{2}-\mu_{0} p \tanh \left(p h_{0}\right)=0$. Then there exists an infinite set $\mathcal{E} \subset \mathbb{R}^{+}$with 0 as a limit point, such that, for any $\varepsilon \in \mathcal{E} \cap\left(0, \varepsilon_{0}\right)$, the system (51) has a solution $X, \mu, h$, with a regular $X$, and estimates $\|X\|=O\left(\varepsilon^{2}\right),\left|\mu-\mu_{0}\right|+\left|h-h_{0}\right|<c \varepsilon$.

Notice that the existence of standing waves is shown on a set of points in the two-dimensional parameter plane, which might be considered as distributed on a curve passing through the point $\left(\mu_{0}, h_{0}\right)$, and that there are infinitely many such points accumulating near $\left(\mu_{0}, h_{0}\right)$. Moreover there are infinitely many points like $\left(\mu_{0}, h_{0}\right)$ in the parameter plane. 
For the infinite depth problem, the dispersion relation is

$$
q^{2}=\mu p
$$

which leads to solutions of the linearized problem under the form

$$
\begin{aligned}
\eta(x, t) & =\cos q t \cos p x, \\
\phi(x, y, t) & =-\frac{q}{p} e^{p y} \sin q t \cos p x, \quad y<0 .
\end{aligned}
$$

This gives solutions as soon as $\mu$ is a positive rational number $r / s$. Then $q=k r$, $p=k^{2} r s, k=1,2, \ldots$ give infinitely many solutions. We shall consider in what follows, the case where $\mu$ is near 1 , since all other cases reduce to this case after a suitable rescaling: dividing the scale of time by $r$, and the length scale by $r s$, multiplies $\mu$ by $s / r$.

The fact that at any rational value of $\mu$, there is an infinite dimensional kernel for the linearized operator $L_{0}$ creates great difficulties, known as "infinitely many resonances". In the paper [8], Amick \& Toland justified the algorithmic approach conjectured by Schwartz \& Whitney [104]. Looking for solutions symmetric under reflexion $x \rightarrow-x$ and even in time $t$, they proved that if one chooses the dominant mode as $\eta(x, t)=\varepsilon \cos t \cos x$, the resonances do not arise at any stage of the computation of the expansion in powers of $\varepsilon$, where $\varepsilon=2 \sqrt{\mu-1}$. The system (51) (without $h$ ) is expressed in the form of an infinite system of coupled ordinary differential equations in the time-periodic spatial Fourier series components of the standing wave. Another way to look at this problem is to use a hamiltonian formulation adapted for spatially periodic solutions, introduced by Zakharov in [127]. Dyachenko and Zakharov [40] have indicated that not only the quadratic terms of the vector field can be removed by a canonical change of variable, but also the resonant cubic terms of the normal form, are such that the truncated system is integrable. This was verified in detail by Craig and Wolfork [30], who showed that the integrability is lost at the next order. Moreover Craig [28] studied the two-mode standing wave solutions of the vector field truncated at order 4. In principle, one can find the formal expansion of all possible standing waves, as they are described below, also by starting with their results.

The infinitely many resonances problem is solved in showing that there are infinitely many formal solutions which may be obtained in expanding in powers of $\varepsilon=2 \sqrt{\mu-1}$, with no difficulty at any order, and where the leading order is given by

$$
\eta(x, t) \sim \varepsilon \sum_{q \in I} \frac{( \pm 1)_{q}}{q^{2}} \cos q^{2} x \cos q t .
$$

The number of basic modes given by $I \subset \mathbb{N}$ may be infinite, and $( \pm 1)_{q}= \pm 1$ (free choice for any $q$ ), so the result of [8] is recovered for $I=\{1\}$. This result justifies previous numerical computations (with hundreds of terms) made with $I=\{1,2\}$ and $I=\{1,2,3\}$ by Bryant and Stiassnie 22. The method of Iooss in [59] uses the formulation of [104], [8], while in [60], the problem is 
directly formulated in the framework of analytic functions. A suitable near identity explicit change of variable allows to modify the system in cancelling the quadratic part, which simplifies greatly the analysis for a Lyapunov-Schmidt method (not using normal forms). The infinite dimensional bifurcation equation has all uncoupled critical modes at leading orders (as might be also found with the results of [30]), and allows to detect all solutions whose power series begins as mentioned in (52). The problem of convergence of such series in a suitable space is still open today. The problem due to infinitely many resonances, in addition to the loss of regularity already present in the finite depth case, leads to serious complications in applying the Nash-Moser theorem. We might however expect that these solutions exist for a restricted set of $\varepsilon \in\left(0, \varepsilon_{0}\right)$, with positive measure.

\section{References}

[1] T.Akylas, F.Dias, R.Grimshaw. The effect of the induced mean flow on solitary waves in deep water. J. Fluid Mech. 355, 317-328, 1998.

[2] C.Amick. Semilinear elliptic eigenvalue problems on an infinite strip with an application to stratified fluids. Ann. Scuola Norm. Sup. Pisa 4, 11, 441499, 1984

[3] C.Amick. On the theory of internal waves of permanent form in fluids of great depth. Trans. AMS, 346, 399-419, 1994.

[4] C.Amick, K.Kirchgässner. A theory of solitary waves in the presence of surface tension. Arch. Rat. Mech. Anal. 105, 1-49, 1989.

[5] C.Amick, J.Toland. On solitary water-waves of finite amplitude. Arch. Rat. Mech. Anal. 76, 9-95, 1981.

[6] C.Amick, J.Toland. On periodic water waves and their convergence to solitary waves in the long-wave limit. Phil. Trans. Roy. Soc. Lond. A 303, 633-669, 1981.

[7] C.Amick, L.Fraenkel, J.Toland. On the Stokes' conjecture for the wave of extreme form. Acta Math. 148, 193-214, 1982.

[8] C.Amick, J.Toland. The semi-analytic theory of standing waves. Proc. Roy. Soc. Lond. A 411, 123-137, 1987.

[9] C.Amick, R.E.L.Turner. A global theory of internal solitary waves in twofluid systems. Trans. AMS, 298, 431-484, 1986.

[10] C.Amick, R.E.L.Turner. Small internal waves in two fluid systems. Arch. Rat. Mech. Anal. 108, 111-139,1989.

[11] V.I.Arnold. Geometrical methods in the theory of ordinary differential equations. Springer-Verlag, New York-Berlin, 1983. 
[12] M. L.Banner, D. H.Peregrine. Wave breaking in deep water. Annu. Rev. Fluid Mech. 25, 373-397, 1993.

[13] M.Barrandon. Résonance 1:2 pour les champs de vecteurs réversibles. C.R.Acad. Sci. Paris I, 334, 7-10, 2002.

[14] J.T.Beale. The existence of solitary water-waves. Comm. Pure Appl. Math. 30, 373-389, 1977.

[15] J.T.Beale. Exact solitary water waves with capillary ripples at infinity. Comm.Pure Appl. Math. 44, 211-257, 1991.

[16] T.B.Benjamin. Internal waves of permanent form in fluids of great depth. J.Fluid Mech. 29, 559-592, 1967.

[17] J.Bona, D.K.Bose, R.E.L.Turner. Finite amplitude steady waves in stratified fluid. J.Math. Pures et Appl. 62, 389-439, 1983.

[18] J.Bona, J.C.Saut. See the paper in the next volume.

[19] T.Bridges. Hamiltonian spatial structure for three-dimensional water waves in a moving frame of reference. J. Nonlinear Science 4, 221-251, 1994.

[20] T.Bridges, G.Derks Dimension breaking of nonlinear elliptic PDEs: satisfying the spectral condition geometrically. Preprint.

[21] T.Bridges, A.Mielke. A proof of the Benjamin-Feir instability. Arch. Rat. Mech. Anal. 133, 145-198, 1995.

[22] P.Bryant, M.Stiassnie. Different forms for nonlinear standing waves in deep water. J. Fluid Mech. 272, 135-156, 1994.

[23] B.Buffoni, A. Champneys, J.Toland. Bifurcation and coalescence of a plethora of homoclinic orbits for a hamiltonian system. J. Dynamics Diff. Equ. 8, 221-281, 1995.

[24] B.Buffoni, M.D.Groves. A multiplicity result for solitary gravity-capillary waves in deep water via critical-point theory. Arch. Rat. Mech. Anal. 146, 183-220, 1999.

[25] A.V.Buryak, A.R.Champneys. On the stability of solitary wave solutions of the fifth-order KdV equation. Phys. Lett. A 233, 58-62, 1997.

[26] A.R.Champneys, J.Toland. Bifurcation of a plethora of multi-modal homoclinic orbits for autonomous Hamiltonian systems. Nonlinearity 6, 665-721, 1993.

[27] A.R.Champneys, J.M.Vanden-Broeck, G.J.Lord. Do true elevation gravitycapillary solitary waves exist? A numerical investigation. J.Fluid Mech. 454, 403-417, 2002. 
[28] W.Craig. Birkhoff normal forms for water waves. Contemp. Math. 200, 57-74, 1996.

[29] W.Craig, D.Nicholls. Travelling two and three dimensional capillary gravity water waves. SIAM J. Math. Anal. 32, 323-359, 2000.

[30] W.Craig, P.Wolfork. An integrable normal form for water waves in infinite depth. Physica D 84, 513-531, 1995.

[31] R.E.Davis, A.Acrivos. Solitary internal waves in deep water. J.Fluid Mech. 29, 593-607, 1967.

[32] F.Dias, M.Hărăguş-Courcelle. On the transition from two-dimensional to three-dimensional water waves. Stud. Appl. Maths 104, 91-127, 2000.

[33] F.Dias, A.Il'ichev. Interfacial waves with free-surface boundary conditions: an approach via a model equation. Physica D 150, 278-300, 2001.

[34] F.Dias, G.Iooss. Capillary-gravity solitary waves with damped oscillations. Physica D 65, 399-423, 1993.

[35] F.Dias, G.Iooss. Capillary-gravity interfacial waves in infinite depth. Eur. J. Mech. B/Fluids 15, 3, 367-393, 1996.

[36] F.Dias, C.Kharif. Nonlinear gravity and capillary-gravity waves. Ann. Rev. Fluid Mech. 31, 301-346, 1999.

[37] F.Dias, D.Menasce, J.M.Vanden-Broeck. Numerical study of capillarygravity solitary waves. Eur. J. Mech. B/Fluids 15, 17-36, 1996.

[38] M.L.Dubreil-Jacotin. Complément à une note antérieure sur les ondes de type permanent dans les liquides hétérogènes. Atti Accad. Lincei, Rend. Cl. Sci. Fis. Mat. Nat. (6), 21, 344-346, 1935.

[39] J.Duncan. Spilling breakers. Annu. Rev. Fluid Mech. 33, 519-547, 2001.

[40] A.I.Dyachenko, V.E.Zakharov. Is free surface hydrodynamics an integrable system? Phys. Letters A 190, 144-148, 1994.

[41] D.M.Farmer, J.D.Smith. Tidal interaction of stratified flow with a sill in Knight Inlet. Deep-Sea Res. 27A, 239-254, 1980.

[42] L.K. Forbes. Surface waves of large amplitude beneath an elastic sheet. Part 1. High-order series solution. J. Fluid Mech. 169, 409-428, 1986.

[43] K.O.Friedrichs, D.H.Hyers. The existence of solitary waves. Comm. Pure Appl. Math. 7, 517-550, 1954.

[44] S.Grilli, P.Guyenne, F.Dias. A fully nonlinear model for three-dimensional overturning waves over arbitrary bottom. Int.J. for Numerical Methods in Fluids 35, 829-867, 2001. 
[45] M.D.Groves. An existence theory for three-dimensional periodic travelling gravity-capillary water waves with bounded transverse profiles. Physica D 152-153, 395-415, 2001.

[46] M.D.Groves, M.Hărăguş, S.M.Sun. A dimension-breaking phenomenon in the theory of steady gravity-capillary water waves. Phil. Trans. Roy. Soc. London (to appear)

[47] M.D.Groves, A.Mielke. A spatial dynamics approach to three-dimensional gravity-capillary steady water waves. Proc. Roy. Soc. Edinburgh A 131 (1), 83-136, 2001.

[48] M.D.Groves, J.F.Toland. On variational formulations for steady water waves. Arch. Rat. Mech. Anal. 137, 203-226, 1997.

[49] J. L.Hammack, D. M.Henderson. Resonant interactions among surface water waves. Annu. Rev. Fluid Mech. 25, 55-97, 1993.

[50] M.Hărăguş-Courcelle, A.Il'ichev. Three-dimensional solitary waves in the presence of additional surface effects. Eur. J. Mech. B/Fluids 17, 5, 739$768,1998$.

[51] M.Hărăguş, K.Kirchgässner. Breaking the dimension of a steady wave: some examples. Nonlinear dynamics and pattern formation in a natural environment. in Pitman Res. Notes Maths 335, 119-129, 1995.

[52] M.Hărăguş, K.Kirchgässner. Breaking the dimension of solitary waves. Progress in partial differential equations: the Metz surveys 4. M.Chipot, I. Shafrir eds. Pitman Res. Notes in Maths, 345, 216-228, 1996.

[53] M.Hărăguş-Courcelle, K.Kirchgässner. Three-dimensional steady capillary - gravity waves. Ergodic Theory, Analysis, and Efficient Simulation of Dynamical systems. B.Fiedler ed., 363-397, Springer Berlin 2001.

[54] M.Hărăguş-Courcelle, R.L.Pego. Travelling waves of the KP equations with transverse modulations. C.R.Acad.Sci. Paris 328, I, 227-232, 1999.

[55] A.Il'ichev, K.Kirchgässner. Nonlinear water waves beneath an elastic icesheet, Preprint Stuttgart University, Bericht 98/19, Sonderforschungsbereich 404, 27 p., 1998.

[56] G.Iooss. A codimension two bifurcation for reversible vector fields. Fields Institute Comm. 4, 201-217, 1995.

[57] G.Iooss. Existence d'orbites homoclines à un équilibre elliptique, pour un système réversible. C.R.Acad.Sci. Paris 324, I, 993-997, 1997.

[58] G.Iooss. Gravity and capillary-gravity periodic travelling waves for two superposed fluid layers, one being of infinite depth. J.Math. Fluid Mech. 1, 24-61, 1999 . 
[59] G.Iooss. Semianalytic theory of standing waves in deep water, for several dominant modes. Proc. Roy. Soc. Lond. A, 455, 3513-3526, 1999.

[60] G.Iooss. On the standing waves problem in deep water. J.Math. Fluid Mech. 4, 155-185, 2002.

[61] G.Iooss. Travelling waves in the Fermi-Pasta-Ulam lattice. Nonlinearity 13, 849-866, 2000.

[62] G.Iooss, M.Adelmeyer. Topics in Bifurcation Theory and Applications. Adv. Series in Nonlinear Dynamics, 3, World Sci. Pub. (2nd ed) 1998.

[63] G.Iooss, K.Kirchgässner. Bifurcation d'ondes solitaires en présence d'une faible tension superficielle. C. R. Acad. Sci. Paris 311, I, 265-268, 1990.

[64] G.Iooss, K.Kirchgässner. Water waves for small surface tension: an approach via normal form. Proc. Roy. Soc. Edinburgh 122A, 267-299, 1992.

[65] G.Iooss, P.Kirrmann. Capillary gravity waves on the free surface of an inviscid fluid of infinite depth. Existence of solitary waves. Arch. Rat. Mech. Anal. 136, 1-19, 1996.

[66] G.Iooss, E.Lombardi, S.M.Sun. Gravity travelling waves for two superposed fluid layers, one being of infinite depth: a new type of bifurcation. Phil. Trans. Roy. Soc. London (to appear).

[67] G.Iooss, J.Los. Bifurcation of spatially quasi-periodic solutions in hydrodynamic stability problems. Nonlinearity 3, 851-871, 1990.

[68] G.Iooss, M.C.Pérouème. Perturbed homoclinic solutions in reversible 1:1 resonance vector fields. J.Diff. Equ. 102, 62-88, 1993.

[69] G.James. Internal travelling waves in the limit of a discontinuous stratified fluid. Arch. Rat. Mech. Anal. 160, 41-90, 2001.

[70] G.James. Small amplitude steady internal waves in stratified fluids. Ann. Univ. Ferrara, Sez VII, Sc. Mat., Vol. XLIII, 65-119, 1997.

[71] M.C.W.Jones. Group invariances, unfoldings and the bifurcation of capillary-gravity waves. Int. J. Bifurcation and Chaos 7, 6, 1243-1266,1997.

[72] T.Kato. Perturbation theory for linear operators. Springer Verlag, 1966.

[73] K.Kirchgässner. Wave solutions of reversible systems and applications. J. Diff. Equ. 45, 113-127, 1982.

[74] K.Kirchgässner. Nonlinearly resonant surface waves and homoclinic bifurcation. Adv. Appl. Mech. 26, 135-181, 1988.

[75] K.Kirchgässner, K.Lankers. Structure of permanent waves in densitystratified media. Meccanica 28, 269-276, 1993. 
[76] P.Kirrmann. Reduktion nichtlinearer elliptischer Systeme in Zylindergebieten unter verwendung von optimaler Regularität in Hölder-Raümen. PhD thesis, Universität Stuttgart, 1991.

[77] Yu.P.Krasovskii. On the theory of steady-state waves of finite amplitude. Comp. Math. Math. Phys. 1, 996-1018, 1961.

[78] K.Lankers, G.Friesecke. Fast, large amplitude solitary waves in the 2D Euler equations for stratified fluids. Nonlinear Analysis TMA, 29, 9, 10611078, 1997.

[79] K.Lankers. Nichtlineare interne Wellen in dichtegesschichteten Flüssigkeiten. PhD Thesis, Univ. Stuttgart, 1997.

[80] M.A.Lavrentiev. A contribution to the theory of long waves (1943); On the theory of long waves (1947). Amer. Math. Soc. Translation, 102, 3-53, 1954.

[81] T.Levi-Civita. Détermination rigoureuse des ondes permanentes d'ampleur finie. Math. Annalen 93, 264-314, 1925.

[82] E.Lombardi. Orbits homoclinic to exponentially small periodic orbits for a class of reversible systems. Application to water waves. Arch. Rat. Mech. Anal. 137, 227-304, 1997.

[83] E.Lombardi. Non-persistence of homoclinic connections for perturbed integrable reversible systems. J. of Dynamics and Diff. Equ. 11, 1, 129-208, 1999.

[84] E.Lombardi. Oscillatory integrals and phenomena beyond all algebraic orders, with applications to homoclinic orbits in reversible systems. Lecture Notes in Maths 1741, Springer, 2000.

[85] E.Lombardi, G.Iooss. Gravity solitary waves with polynomial decay to exponentially small ripples at infinity. Ann. IHP - Anal. Non Linéaire (to appear).

[86] M.S.Longuet-Higgins, X.Zhang. Experiments on capillary-gravity waves of solitary type on deep water. Phys. Fluids 9, 1963-1968, 1997.

[87] A.M.Lyapounov. Problème général de la stabilité du mouvement. Ann. Fac. Sci. Toulouse 9, 1907 (russian original 1895).

[88] R.S.MacKay, P.G.Saffman. Stability of water waves. Proc. Roy. Soc. London A 406, 115-125, 1986.

[89] A.P.Metha, B.R.Sutherland. Interfacial gravity currents: Part II - wave excitation. Phys. Fluids, submitted.

[90] H.Michallet, E.Barthélemy. Experimental study of interfacial solitary waves. J.Fluid Mech. 366, 159-177, 1998. 
[91] H.Michallet, F.Dias. Numerical study of generalized interfacial solitary waves. Phys. Fluids 11, 1502-1511, 1999.

[92] A.Mielke. Reduction of quasilinear elliptic equations in cylindrical domains with applications. Math. Meth. Appl. Sci. 10, 51-66, 1988.

[93] A.Mielke. Homoclinic and heteroclinic solutions in two-phase flow. Adv. Series in Nonlinear Dyn., Proc. IUTAM/ISIMM Symp. on Structure and Dynamics of Nonlinear waves in fluids. World Sci. 7, 353-362, 1995.

[94] A.I.Nekrasov. On waves of permanent type. Izv. Ivanovo-Voznesensk. Politekhn. Inst., 3, 52-65, 1921.

[95] H.Ono. Algebraic solitary waves in stratified fluids. J.Phys. Soc. Japan 39, 1082-1091, 1975.

[96] E.Părău, F.Dias. Interfacial periodic waves of permanent form with freesurface boundary conditions. J.Fluid Mech. 437, 325-336, 2001.

[97] E.Părău, F.Dias. Nonlinear effects in the response of a floating ice plate to a moving load. J. Fluid Mech. 460, 281-305, 2002.

[98] M.Perlin, W.W.Schultz. Capillary effects on surface waves. Annu. Rev. Fluid Mech. 32, 241-274, 2000.

[99] P.I.Plotnikov, J.F.Toland. Nash-Moser theory for standing water waves. Arch. Rat. Mech. Anal. 159, 1-83, 2001.

[100] J.Reeder, M.Shinbrot. Three-dimensional, nonlinear wave interaction in water of constant depth. Nonlinear Analysis TMA 5, 303-323, 1981.

[101] R.L.Sachs. On the existence of small amplitude solitary waves with strong surface tension. J. Diff. Equations, 90, 1, 31-51, 1991.

[102] R.Scardovelli, S.Zaleski. Direct numerical simulation of free-surface and interfacial flow. Annu. Rev. Fluid Mech. 31, 567-603, 1999.

[103] G.Schneider, C.E. Wayne. The long wave limit for the water-wave problem -I. The case of zero surface tension. Comm. Pure Appl. Math. 53, 1475$1535,2000$.

[104] L.Schwartz, A.Whitney. A semi-analytic solution for nonlinear standing waves in deep water. J.Fluid Mech. 107, 147-171, 1981.

[105] Shore Protection Manual. 4th ed., U.S. Army Corps Engrs., Coastal Eng. Res. Center, U.S. Govt. Printing Office, Washington, D.C., 1984.

[106] V.A.Squire, R.J.Hosking, A.D.Kerr, P.J.Langhorne. Moving loads on ice plates. Solids Mechanics and its Applications, 45, Kluwer Acad. Pub. 1996.

[107] V.A.Squire, W.H.Robinson, P.J. Langhorne, T.G.Haskell. Vehicles and aircraft on floating ice. Nature 333, 159-161, 1988. 
[108] G.G.Stokes. On the theory of oscillatory waves. Camb. Phil. Soc. Trans. 8, 441-455, 1847.

[109] S.M.Sun. Existence of a generalized solitary wave solution for water with positive Bond number less than 1/3. J.Math. Anal.Appl. 156, 471-504, 1991.

[110] S.M.Sun. Non existence of truly solitary waves in water with small surface tension. Proc. Roy. Soc. London A 455, 2191-2228, 1999.

[111] S.M.Sun. Some analytical properties of capillary-gravity waves in two-fluid flows of infinite depth. Proc. Roy. Soc. London A 453, 1153-1175, 1997.

[112] S.M.Sun. Existence of solitary internal waves in a two-layer fluid of infinite depth. Nonlinear Analysis 30, 8, 5481-5490, 1997.

[113] S.M.Sun, M.C.Shen. Exponentially small estimate for the amplitude of capillary ripples of a generalized solitary wave. J.Math. Anal.Appl. 172, 533-566, 1993.

[114] S.M.Sun, M.C.Shen. Exact theory of generalized solitary waves in a twolayer liquid in the absence of surface tension. J. Math. Anal. Appl. 180, 245-274, 1993.

[115] T.Takizawa. Field studies on response of a floating sea ice sheet to a steadily moving load. Contributions from the Institute of Low temperature Science A 36, 31-76, 1987.

[116] J.F. Toland. Stokes waves. Topological Meth. in Nonlinear Anal. 7 and 8, 1-48; 412-414, 1996 and 1997.

[117] W.Tsai, D. K. P. Yue. Computation of nonlinear free-surface flows. Annu. Rev. Fluid Mech. 28, 249-278, 1996.

[118] R.E.L.Turner. Internal waves in fluids with rapidly varying density. Ann. Scuola Norm. Sup. Pisa Cl. Sci. 8, 513-573, 1981.

[119] A.Vanderbauwhede, G.Iooss. Center manifold theory in infinite dimensions. Dynamics Reported, 1 New series, 125-163, 1992.

[120] J. V.Wehausen, E. V. Laitone. Surface waves. Handbuch der Physik, Ed. S. Flügge, Vol. 9, Springer-Verlag, 446-778, 1960.

[121] P.D.Woods, A.R.Champneys. Heteroclinic tangles and homoclinic snaking in the unfolding of a degenerate reversible Hamiltonian-Hopf bifurcation. Physica D 129, 147-170, 1999.

[122] S.Wu. Well-posedness in Sobolev spaces of the full water wave problem in 2-D. Invent. Math. 130, 39-72, 1997. 
[123] S.Wu. Well-posedness in Sobolev spaces of the full water wave problem in 3-D. J. Am. Math. Soc. 12, 2, 445-495, 1999.

[124] T.S.Yang, T.R.Akylas. Weakly nonlocal gravity-capillary solitary waves. Phys. Fluids 8, 1506-1514, 1996.

[125] T.S.Yang, T.R.Akylas. On asymmetric gravity-capillary solitary waves. J.Fluid Mech. 330, 215-232, 1997.

[126] X. Zhang. Capillary-gravity and capillary waves generated in a wind wave tank: observations and theories. J. Fluid Mech. 289, 51-82, 1995

[127] V.E.Zakharov. Stability of periodic waves of finite amplitude on the surface of deep fluid. J. Appl. Mech. Tech. Physics 2, 190-194, 1968. 